Research Article

\title{
Vibration Characteristics of Rotating Mistuned Bladed Disks considering the Coriolis Force, Spin Softening, and Stress Stiffening Effects
}

\author{
Shuai Wang $\mathbb{D},{ }^{1,2}$ Chuanxing $\mathrm{Bi}^{1,2}$ Bin $\mathrm{Zi}^{2}$, and Changjun Zheng $\mathbb{D}^{1,2}$ \\ ${ }^{1}$ Institute of Sound and Vibration Research, Hefei University of Technology, Hefei 230009, China \\ ${ }^{2}$ School of Mechanical Engineering, Hefei University of Technology, Hefei 230009, China \\ Correspondence should be addressed to Changjun Zheng; cjzheng@hfut.edu.cn
}

Received 24 April 2019; Revised 29 July 2019; Accepted 21 October 2019; Published 12 November 2019

Academic Editor: Athanasios Chasalevris

Copyright (c) 2019 Shuai Wang et al. This is an open access article distributed under the Creative Commons Attribution License, which permits unrestricted use, distribution, and reproduction in any medium, provided the original work is properly cited.

Bladed disks of engine rotors usually operate at harsh conditions of high rotating speeds, which may lead to nonnegligible rotordynamic effects, including Coriolis force, spin softening, and stress stiffening effects. These effects on the vibration of mistuned bladed disks are seldom discussed in available investigations. In this paper, the vibration characteristics of rotating mistuned bladed disks are addressed by considering these rotordynamic effects. First, finite element (FE) models of bladed disks are used to obtain the governing equations of motion, and an efficient method for getting the stress stiffening matrix of sector model is developed. Then, the effective component-mode mistuning method (CMM) is employed to create compact, yet accurate, reduced-order models (ROMs). Finally, the models are validated and used to study the influences of Coriolis force, spin softening, and stress stiffening effects on the vibration of bladed disks with frequency mistuning factors. Numerical results show that these rotordynamic effects could significantly affect the vibrations of mistuning bladed disks, especially in the ranges of high speed, and should be carefully considered during analysis.

\section{Introduction}

The vibration and high-cycle fatigue issues of bladed disks have always been the focuses of researchers and engineers in the past few decades. Mistuning is widely deemed as the main cause for high-cycle fatigue in bladed disks, whereas it is unavoidable in practical applications due to the inevitable manufacturing tolerance and in-service wear and tear. As a consequence, the adverse effects of mistuning on the vibration of bladed disks and effective methods for relieving these effects have attracted enormous attentions in the past decades, which have been comprehensively reviewed in [1-3]. Although abundant achievements have been gained, mistuning is still an unresolved problem for turbomachinery designers, as was stated by Ewins and Chan [4]. Nevertheless, it is believable that accurately obtaining the vibration characteristics of mistuned bladed disks with more properly considering their operating conditions could be an important basis for settling this issue.
The realistic operating conditions of bladed disks may contain complex coupling between structure, flow field, and rotordynamic effects. In the early stage of studies, most investigations focused either on the structural vibrations of mistuned bladed disks [5-9] or on the beneficial effects of mistuning to flutter [10-13]. In recent years, a number of investigations have been published to address the effects of aerodynamic damping [14-19] and to develop effective reduced-order models for quantitative vibration analysis. In obtaining reduced-order models of mistuned bladed disks, the substructure techniques and component-mode synthesis methods [20-22] are commonly employed in the early stage. In contrast, Yang and Griffin [23] presented a novel way of including mistuning parameter in reduced-order models by representing the modes of the mistuned system in terms of a subset of nominal system modes. On this basis, Feiner and Griffin [24] developed a more effective reduced-order model of mistuned bladed disks by employing only a single family 
of modes. Lim et al. [25] presented a novel component-based modelling framework by partitioning mistuned bladed disk into a tuned bladed disk component and virtual blade mistuning components. Sternchüss [26] introduced a model order reduction technique which can handle parametric models depending on rotation speed. Beck et al. [27] developed two new geometric mistuning reduced-order models synthesized from an IBR composed of a tuned disk and geometrically perturbed airfoil by using geometrically mistuned airfoil modes in the component-mode framework. Vargiu et al. [28] extended the Component-Mode Mistuning technique to allow for the introduction of a sector frequency mistuning pattern. D'Souza et al. [29] developed compact ROMs for mistuned multistage bladed disk systems with aerodynamic effects included.

Compared with the large number of investigations on the vibration of mistuned bladed disks, relatively few papers were published to address the influences of the rotordynamic effects. Actually, bladed disks of engine rotors tend to operate at rotating conditions with high speeds, which may lead to significant rotordynamic effects, including Coriolis force, spin softening, and stress stiffening effects. Among the small number of investigations, only the Coriolis forces were taken into consideration. Huang and Kuang [30] investigated the effects of Coriolis forces on the mode localization of a weakly coupled mistuned shrouded bladed disk and concluded that the Coriolis force may enhance the localization phenomenon. Nikolic et al. [31] addressed the mutual interaction of Coriolis forces and mistuning on the vibration characteristics of bladed disks and found that the Coriolis force may affect the vibrations of bladed disks at some designs. Kan et al. [32] studied the effect of Coriolis force on forced response of intentionally mistuned bladed disk. Although the effects of Coriolis force have been discussed, the influences of the spin softening and stress stiffening effects on the vibration of mistuned bladed disks are not clear. How these effects affect the system's forced response and the statistical characteristics of response and to what extent remain to be investigated.

In this paper, the vibration characteristics of rotating mistuned bladed disks are addressed by taking a full consideration of the Coriolis force, spin softening, and stress stiffening effects. Although both the aerodynamic and rotordynamic effects should be taken into account in order to accurately reflect the vibrations of rotating bladed disks, this paper concentrates particularly on the influences of the rotordynamic effects. For the sake of quantitative modelling and analysis, finite element models of bladed disks are employed for obtaining the governing equations of motion. In order to remarkably reduce the computational requirement during analysis, the effective CMM method is employed to create compact and accurate ROMs. On this basis, the reduced-order models are validated and then used to investigate the influences of the rotordynamic effects on the vibration characteristics of bladed disks with frequency mistuning factors. This paper is organized as follows. Section 2 contains the mathematical formulations for dynamic modelling and model order reduction. In Section 3, validation of the obtained reduced-order model is presented.
Section 4 contains the numerical results. Conclusions are given in Section 5.

\section{Mathematical Formulation}

2.1. Governing Equations of Motion. According to the rotordynamic analysis method [33], the governing equations of motion of a mistuned bladed disk rotating at a constant angular velocity $\Omega$ can be expressed as

$$
\mathbf{M u}(t)+\left[\mathbf{C}_{d}+\Omega \mathbf{C}_{\text {cor }}\right] \dot{\mathbf{u}}(t)+\left[\mathbf{K}_{e}-\Omega^{2} \mathbf{K}_{p}+\mathbf{K}_{s}(\Omega)+\mathbf{K}_{\delta}\right] \mathbf{u}(t)=\mathbf{f}(t),
$$

where $\mathbf{M}, \mathbf{C}_{d}$, and $\mathbf{K}_{e}$ denote the mass, damping, and elastic stiffness matrices of the system; $\mathbf{u}(t)$ is the vector of response; $\Omega \mathrm{C}_{\text {cor }}$ is a skew-symmetric speed-dependent matrix due to Coriolis forces; $\Omega^{2} \mathbf{K}_{p}$ denotes a speed-dependent spin softening matrix due to the rotation of structure; $\mathbf{K}_{s}(\Omega)$ is a speed-dependent stress stiffening matrix due to centrifugal forces; $\mathbf{K}_{\delta}$ represents the stiffness matrices due to mistuning; and $\mathbf{f}(t)$ denotes the vector of applied external force.

For bladed disks, the external force derives from the aerodynamic pressures acted on the blades, which is often assumed to be a traveling wave excitation. The excitations can be classified as backward and forward ones according to their traveling directions $[16,31]$. Backward traveling wave excitation travels opposite to the rotation direction and commonly exists in practical applications. Forward traveling wave excitation has identical traveling direction as the rotation direction and may exist at some circumstances. In this paper, the external force is assumed to be a traveling wave excitation, where the force on the $n$th blade has the form as

$$
\mathbf{f}_{n}(t)=\mathbf{f}_{0} \cos \left[C\left(\Omega t \pm \varphi_{n}\right)\right], \quad n=1,2, \ldots, N,
$$

where $\mathbf{f}_{0}$ is the amplitude vector of external force; $C$ denotes the engine order (EO) of excitation; and $\varphi_{n}$ denotes the phase angle and is defined as $\varphi_{n}=(n-1) 2 \pi / N$, where $N$ is the number of sectors. For a backward traveling wave excitation, the sign before $\varphi_{n}$ in equation (2) takes a minus sign, and vice versa.

Compared with the Coriolis matrix $\Omega \mathrm{C}_{\text {cor }}$ and the spin softening matrix $\Omega^{2} \mathbf{K}_{p}$ in equation (1), which are explicitly dependent on the rotation speed, the stress stiffening matrix $\mathbf{K}_{s}(\Omega)$ is an implicitly dependent matrix regarding $\Omega$. Namely, the $\mathbf{K}_{s}(\Omega)$ matrices at two different speeds are not directly and explicitly related. It is difficult to deal with such matrix during analysis, as a list of $\mathbf{K}_{s}(\Omega)$ at different speeds should be obtained beforehand when the forced response within a certain range of speed is of interest. With respect to this issue, the parametrization method developed by Sternchüss [26] is employed herein. According to the Sternchüss's method, the overall stiffness matrix $\mathbf{K}(\Omega)=$ $\left[\mathbf{K}_{e}-\Omega^{2} \mathbf{K}_{p}+\mathbf{K}_{s}(\Omega)\right]$ can be approximately parametrized regarding rotating speed $\Omega$ as

$$
\mathbf{K}(\Omega)=\mathbf{K}_{\Omega, 0}+\Omega^{2} \mathbf{K}_{\Omega, 1}+\Omega^{4} \mathbf{K}_{\Omega, 2}
$$

where $\mathbf{K}_{\Omega, 0}, \mathbf{K}_{\Omega, 1}$, and $\mathbf{K}_{\Omega, 2}$ are the coefficient matrices represented by the stiffness matrices at three different 
rotating speeds $\Omega_{0}, \Omega_{1}$, and $\Omega_{2}$. For a particular case with $\Omega_{0}=0, \Omega_{1}=\Omega_{\max } / 2$, and $\Omega_{2}=\Omega_{\max }$, where $\Omega_{\max }$ denotes the maximum speed of interest, the coefficient matrices in equation (3) can be simplified as

$$
\begin{aligned}
& \mathbf{K}_{\Omega, 0}=\mathbf{K}(0), \\
& \mathbf{K}_{\Omega, 1}=\frac{1}{3 \Omega_{\max }^{2}}\left[16 \mathbf{K}\left(\frac{\Omega_{\max }}{2}\right)-\mathbf{K}\left(\Omega_{\max }\right)-15 \mathbf{K}(0)\right], \\
& \mathbf{K}_{\Omega, 2}=\frac{4}{3 \Omega_{\max }^{4}}\left[\mathbf{K}\left(\Omega_{\max }\right)-4 \mathbf{K}\left(\frac{\Omega_{\max }}{2}\right)+3 \mathbf{K}(0)\right] .
\end{aligned}
$$

Such parametrization brings great convenience for analysis, by which only three cases of stiffness matrices are needed to explicitly obtain the stiffness matrix at any rotating speed.

In order to illustrate the different influences of Coriolis force, spin softening, and stress stiffening effects on the vibration of mistuned bladed disks, these three effects are considered in sequence in subsequent analysis. Actually, these three effects exist simultaneously in rotating structures and should all be taken into account to obtain accurate vibration characteristics. Herein, these effects are considered in turn to effectively reveal their diverse influences on the vibration of mistuned bladed disks. As a consequence, there are four different cases of analysis, which are cases without any rotordynamic effects, considering the Coriolis force, considering the Coriolis forces and spin softening effect, and considering all rotordynamic effects, respectively. By denoting the overall damping matrix as $\mathbf{C}(\Omega)$, the overall damping and stiffness matrices of the four cases can be expressed as

$$
\begin{aligned}
& \left\{\begin{array}{l}
\mathbf{C}(\Omega)=\mathbf{C}_{d}, \\
\mathbf{K}(\Omega)=\mathbf{K}_{e}+\mathbf{K}^{\delta},
\end{array}\right. \\
& \left\{\begin{array}{l}
\mathbf{C}(\Omega)=\mathbf{C}_{d}+\Omega \mathbf{C}_{\text {cor }}, \\
\mathbf{K}(\Omega)=\mathbf{K}_{e}+\mathbf{K}^{\delta},
\end{array} \quad\right. \text { case : COR, } \\
& \left\{\begin{array}{l}
\mathbf{C}(\Omega)=\mathbf{C}_{d}+\Omega \mathbf{C}_{\text {cor }}, \\
\mathbf{K}(\Omega)=\mathbf{K}_{e}-\Omega^{2} \mathbf{K}_{p}+\mathbf{K}^{\delta},
\end{array} \quad\right. \text { case : SOF, } \\
& \left\{\begin{array}{l}
\mathbf{C}(\Omega)=\mathbf{C}_{d}+\Omega \mathbf{C}_{\text {cor }}, \\
\mathbf{K}(\Omega)=\mathbf{K}_{e}-\Omega^{2} \mathbf{K}_{p}+\mathbf{K}_{s}(\Omega)+\mathbf{K}^{\delta},
\end{array}\right. \text { case : STF. }
\end{aligned}
$$

The abbreviations "STA," "COR," "SOF," and "STF" in equation (5) are employed herein for simplifying the description in subsequent analysis. The STA case is the most common circumstances in available investigations. The COR, SOF, and STF cases denote those with successive additions of Coriolis force, spin softening, and stress stiffening effects. As the analyses in this paper are all conducted in steady conditions without considering of acceleration, the mass matrices of the four cases are all the same.

Generally, the sizes of the full-order matrices in equation (1) are too large to deal with during analysis. Cyclic-symmetric analysis is often applied by employing much smaller matrices of a single sector model. The matrices regarding mass, damping, elastic stiffness, Coriolis force, and spin softening effect can be easily obtained by the finite element model of a single sector. Nevertheless, the stress stiffness matrix can hardly be acquired by such approach, as it depends on the stresses of the structures during statics analysis under the action of centrifugal forces. During statics analysis, cyclicsymmetric boundary conditions should be applied to accurately reflect the realistic displacements of a bladed disk. Such processing will result in the combination of the stiffness coefficients with respect to the nodes on the boundaries. Regarding this issue, an efficient method using a three-sector model for computing the stress stiffening matrix is developed in this paper and presented, for simplicity, in Appendix.

2.2. Reduced-Order Modelling. FE models of bladed disks tend to possess huge sizes, which greatly affect the computational efficiency, especially for statistical analysis. Thus, a number of model order reduction techniques have been developed in recent years to deal with this issue. In this paper, the effective CMM method proposed by Lim at al. [25] is employed to create compact and accurate reducedorder models for mistuned bladed disks. The core idea of the CMM method contains two aspects, which are representing the mistuned normal modes by tuned normal modes and projecting the stiffness mistuning matrices to the normal modes of tuned blade cantilevered at its root. In [25], the real form cyclic analysis is employed. Regarding the cases considering the rotordynamic effects in this paper, which contain complex damping matrices, the complex form cyclic analysis is employed instead to simplify the deduction. The procedures of the CMM method are briefly reviewed in this section.

First, the response $\mathbf{u}(t)$ of the mistuned system is represented by the superposition of the modes of the corresponding tuned counterpart as

$$
\mathbf{u}(t)=\boldsymbol{\Phi} \mathbf{\eta}(t),
$$

where $\boldsymbol{\eta}(t)$ denotes the modal coordinates and $\boldsymbol{\Phi}$ is the mode matrices of tuned bladed disk and

$$
\boldsymbol{\Phi}=\left(\mathbf{E} \otimes \mathbf{I}_{s}\right) \underset{n=1,2, \cdots, N}{\operatorname{Bdiag}}\left(\widetilde{\boldsymbol{\Phi}}_{n}^{s}\right),
$$

where $\mathbf{E}$ is the complex Fourier matrix of dimension $N$ with the coefficient $e_{u v}=e^{j \alpha(u-1)(v-1)} / \sqrt{N}$ for $u, v=1,2, \ldots, N$, where $j=\sqrt{-1}$ and $\alpha=2 \pi / N ; \otimes$ denotes the Kronecker product; $\mathbf{I}_{s}$ is an identity matrix of dimention $N_{s}$, where $N_{s}$ denotes the number of degrees of freedom of the sector model; and Bdiag $\left(\widetilde{\boldsymbol{\Phi}}_{n}^{s}\right)$ denotes a pseudo-block diagonal matrix with $\widetilde{\Phi}_{n}^{s}$ for $n=1,2, \ldots, N$ along its diagonal blocks, where $\widetilde{\Phi}_{n}^{s}$ is the mode matrix of tuned sector model obtained by the following eigen equation:

$$
\left[\widetilde{\mathbf{K}}_{e, n}^{s}-\lambda^{2} \widetilde{\mathbf{M}}_{n}^{s}\right] \widetilde{\boldsymbol{\Phi}}_{n}^{s}=\mathbf{0}, \quad n=1,2, \ldots, N
$$

where $\lambda$ denotes the eigenvalue and 


$$
\begin{aligned}
\tilde{\mathbf{M}}_{n}^{s} & =\mathbf{M}_{0}^{s}+\mathbf{M}_{1}^{s} e^{j(n-1) \theta}+\left(\mathbf{M}_{1}^{s}\right)^{\mathrm{T}} e^{-j(n-1) \theta}, \\
\widetilde{\mathbf{K}}_{e, n}^{s} & =\mathbf{K}_{e, 0}^{s}+\mathbf{K}_{e, 1}^{s} e^{j(n-1) \theta}+\left(\mathbf{K}_{e, 1}^{s}\right)^{\mathrm{T}} e^{-j(n-1) \theta},
\end{aligned}
$$

where

$$
\begin{aligned}
& \mathbf{M}_{0}^{s}=\left[\begin{array}{cc}
\mathbf{M}_{a a}^{s}+\mathbf{M}_{b b}^{s} & \mathbf{M}_{a i}^{s} \\
\mathbf{M}_{i a}^{s} & \mathbf{M}_{i i}^{s}
\end{array}\right], \\
& \mathbf{M}_{1}^{s}=\left[\begin{array}{ll}
\mathbf{M}_{a b}^{s} & \mathbf{0} \\
\mathbf{M}_{i b}^{s} & \mathbf{0}
\end{array}\right] \text {, } \\
& \mathbf{K}_{0}^{s}=\left[\begin{array}{cc}
\mathbf{K}_{a a}^{s}+\mathbf{K}_{b b}^{s} & \mathbf{K}_{a i}^{s} \\
\mathbf{K}_{i a}^{s} & \mathbf{K}_{i i}^{s}
\end{array}\right] \text {, } \\
& \mathbf{K}_{1}^{s}=\left[\begin{array}{ll}
\mathbf{K}_{a b}^{s} & \mathbf{0} \\
\mathbf{K}_{i b}^{s} & \mathbf{0}
\end{array}\right],
\end{aligned}
$$

where the subscript symbols " $a$," " $i$, , and " $b$ " represent the upper boundary, interior part, and lower boundary of a sector model, respectively.

Then, in order to include the mistuning factors, the stiffness mistuning matrix $\mathbf{K}_{\delta}$ is projected to the normal modes of a tuned blade cantilevered at its root. It is realized by projecting the blade parts of $\Phi$ to the normal modes and constraint modes of cantilevered blade as

$$
\boldsymbol{\Phi}_{B}=\left(\mathbf{I}_{N} \otimes \mathbf{U}_{C B}\right) \mathbf{q}_{B},
$$

where $\boldsymbol{\Phi}_{B}$ denotes the blade parts of $\boldsymbol{\Phi} ; \mathbf{I}_{N}$ is an identity matrix of dimension $N ; \mathbf{q}_{B}$ is the participation factor vector of the normal modes and constraint modes; and $\mathbf{U}_{C B}$ is the transformation matrix of the well-known Craig-Bampton fixed-interface component-mode synthesis method and

$$
\mathbf{U}_{C B}=\left[\begin{array}{cc}
\boldsymbol{\Phi}_{o}^{B} & \Psi_{o}^{B} \\
\mathbf{0} & \mathbf{I}_{b}^{B}
\end{array}\right],
$$

where $\boldsymbol{\Phi}_{o}^{B}$ and $\boldsymbol{\Psi}_{o}^{B}$ denote the normal mode and constraint mode matrices of the cantilevered blade, respectively, and $\mathbf{I}_{b}^{B}$ is an identity matrix of dimension $N_{b}^{B}$, where $N_{b}^{B}$ is the number of degrees of freedom of the fixed-interface nodes.

By substituting equation (6) into equation (1), the governing equations of the reduced-order model can be obtained as

$$
\tilde{\mathbf{M}} \ddot{\mathbf{\eta}}(t)+\left[\widetilde{\mathbf{C}}_{d}+\Omega \widetilde{\mathbf{C}}_{c o r}\right] \dot{\boldsymbol{\eta}}(t)+\left[\widetilde{\mathbf{K}}_{e}-\Omega^{2} \widetilde{\mathbf{K}}_{p}+\widetilde{\mathbf{K}}_{s}(\Omega)+\widetilde{\mathbf{K}}_{\delta}\right] \boldsymbol{\eta}(t)=\widetilde{\mathbf{f}}(t),
$$

where

$$
\begin{gathered}
\tilde{\mathbf{M}}=\boldsymbol{\Phi}^{\mathrm{T}} \mathbf{M} \boldsymbol{\Phi}=\underset{n=1,2, \ldots, N}{\text { Bdiag }}\left[\left(\widetilde{\boldsymbol{\Phi}}_{n}^{s}\right)^{\mathrm{T}} \widetilde{\mathbf{M}}_{n}^{s} \widetilde{\boldsymbol{\Phi}}_{n}^{s}\right], \\
\widetilde{\mathbf{C}}_{d}=\boldsymbol{\Phi}^{\mathrm{T}} \mathbf{C}_{d} \boldsymbol{\Phi}=\underset{n=1,2, \ldots, N}{\text { Bdiag }}\left[\left(\widetilde{\boldsymbol{\Phi}}_{n}^{s}\right)^{\mathrm{T}} \widetilde{\mathbf{C}}_{n}^{s} \widetilde{\boldsymbol{\Phi}}_{n}^{s}\right], \\
\widetilde{\mathbf{C}}_{\text {cor }}=\boldsymbol{\Phi}^{\mathrm{T}} \mathbf{C}_{\text {cor }} \boldsymbol{\Phi}=\underset{n=1,2, \ldots, N}{\operatorname{Bdiag}}\left[\left(\widetilde{\boldsymbol{\Phi}}_{n}^{s}\right)^{\mathrm{T}} \widetilde{\mathbf{C}}_{c o r}^{s} \widetilde{\boldsymbol{\Phi}}_{n}^{s}\right], \\
\widetilde{\mathbf{K}}_{e}=\boldsymbol{\Phi}^{\mathrm{T}} \mathbf{K}_{e} \boldsymbol{\Phi}=\underset{n=1,2, \ldots, N}{\operatorname{Bdiag}}\left[\left(\widetilde{\boldsymbol{\Phi}}_{n}^{s}\right)^{\mathrm{T}} \widetilde{\mathbf{K}}_{e, n}^{s} \widetilde{\boldsymbol{\Phi}}_{n}^{s}\right], \\
\widetilde{\mathbf{K}}_{p}=\boldsymbol{\Phi}^{\mathrm{T}} \mathbf{K}_{p} \boldsymbol{\Phi}=\underset{n=1,2, \ldots, N}{\operatorname{Bdiag}}\left[\left(\widetilde{\boldsymbol{\Phi}}_{n}^{s}\right)^{\mathrm{T}} \widetilde{\mathbf{K}}_{p, n}^{s} \widetilde{\boldsymbol{\Phi}}_{n}^{s}\right], \\
\widetilde{\mathbf{K}}_{s}(\Omega)=\boldsymbol{\Phi}^{\mathrm{T}} \mathbf{K}_{s}(\Omega) \boldsymbol{\Phi}=\underset{n=1,2, \ldots, N}{\operatorname{Bdiag}}\left[\left(\widetilde{\boldsymbol{\Phi}}_{n}^{s}\right)^{\mathrm{T}} \widetilde{\mathbf{K}}_{s, n}^{s} \widetilde{\boldsymbol{\Phi}}_{n}^{s}\right], \\
\widetilde{\mathbf{f}}(t)=\boldsymbol{\Phi}^{\mathrm{T}} \mathbf{f}(t)=\underset{n=1,2, \ldots, N}{\operatorname{Bdiag}}\left[\left(\widetilde{\boldsymbol{\Phi}}_{n}^{s}\right)^{\mathrm{T}}\right]\left(\mathbf{E}^{*} \otimes \mathbf{I}_{s}\right) \mathbf{f}(t),
\end{gathered}
$$

where $\widetilde{\mathbf{C}}_{n}^{s}, \widetilde{\mathbf{C}}_{\text {cor }}^{s}, \widetilde{\mathbf{K}}_{p, n}^{s}$, and $\widetilde{\mathbf{K}}_{s, n}^{s}$ are the matrices obtained by the cyclic sector model and have similar forms as $\widetilde{\mathbf{M}}_{n}^{s}$ and $\widetilde{\mathbf{K}}_{e, n}^{s}$ in equation (9) and $\mathbf{E}^{*}$ denotes the conjugate transpose of $\mathbf{E}$. If mistuning is assumed to exist only in the blade, $\widetilde{\mathbf{K}}_{\delta}=$ $\boldsymbol{\Phi}^{\mathrm{T}} \mathbf{K}_{\delta} \boldsymbol{\Phi}$ can be expressed as

$$
\widetilde{\mathbf{K}}_{\delta}=\left(\boldsymbol{\Phi}_{B}\right)^{\mathrm{T}} \mathbf{K}_{B, \delta} \boldsymbol{\Phi}_{B}=\sum_{n=1}^{N}\left[\left(\mathbf{q}_{B, n}\right)^{\mathrm{T}}\left(\mathbf{U}_{C B}\right)^{\mathrm{T}} \mathbf{K}_{B, \delta, n} \mathbf{U}_{C B} \mathbf{q}_{B, n}\right],
$$

where $\mathbf{q}_{B, n}$ and $\mathbf{K}_{B, \delta, n}$ denote the components of $\mathbf{q}_{B}$ and $\mathbf{K}_{B, \delta}$ corresponding to the $n$th blade.

Furthermore, if proportional mistuning is assumed, namely, by introducing small random variation in the Young's modulus of each blade

$$
E_{n}=\left(1+\delta_{n}\right) E_{0},
$$

where $E_{n}$ is Young's modulus of the $n$th mistuned blade; $E_{0}$ is the nominal Young's modulus; $\delta_{n}$ is a random variable; and $\left(\mathbf{U}_{C B}\right)^{\mathrm{T}} \mathbf{K}_{B, \delta, n} \mathbf{U}_{C B}$ can be expressed as $\delta_{n}\left(\mathbf{U}_{C B}\right)^{\mathrm{T}} \mathbf{K}_{o}^{B} \mathbf{U}_{C B}$, where $\mathbf{K}_{o}^{B}$ denotes the stiffness matrix of tuned cantilevered blade. Then, $\widetilde{\mathbf{K}}_{\delta}$ can be simply expressed as

$$
\widetilde{\mathbf{K}}_{\delta}=\boldsymbol{\delta}\left(\mathbf{q}_{B}\right)^{\mathrm{T}}\left(\mathbf{I}_{N} \otimes \widetilde{\mathbf{K}}_{o}^{B}\right) \mathbf{q}_{B},
$$

where $\boldsymbol{\delta}=\left[\begin{array}{llll}\delta_{1} & \delta_{2} & \cdots & \delta_{N}\end{array}\right]$ and $\widetilde{\mathbf{K}}_{o}^{B}=\left(\mathbf{U}_{C B}\right)^{\mathrm{T}} \mathbf{K}_{o}^{B} \mathbf{U}_{C B}$. As $\left(\mathbf{q}_{B}\right)^{\mathrm{T}}\left(\mathbf{I}_{N} \otimes \widetilde{\mathbf{K}}_{o}^{B}\right) \mathbf{q}_{B}$ is not dependent on the applied mistuning patterns, the mistuning matrix $\widetilde{\mathbf{K}}_{\delta}$ can be efficiently obtained, which can bring convenience for forced response analysis, especially for statistical analysis.

Regarding the external force $\widetilde{\mathbf{f}}(t)$ in equation (13), it can also be expanded and simplified. By transferring the force $\mathbf{f}_{n}(t)$ in equation (2) into exponential form as $\mathbf{f}_{n}(t)=\mathbf{f}_{0} e^{j C\left(\Omega t \pm \varphi_{n}\right)}, n=1,2, \ldots, N$, the overall external force $\mathbf{f}(t)$ can be expressed as $\mathbf{f}(t)=e^{j C \Omega t}\left[\mathbf{f}_{0}^{\mathrm{T}} e^{ \pm j C \varphi_{1}}\right.$

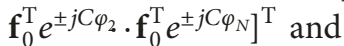




$$
\mathbf{f}(t)= \begin{cases}\sqrt{N} e^{j C \Omega t}\left(\mathbf{e}_{C+1} \otimes \mathbf{f}_{0}\right), & \text { for forward travelling wave excitation, } \\ \sqrt{N} e^{j C \Omega t}\left(\mathbf{e}_{N-C+1} \otimes \mathbf{f}_{0}\right), & \text { for backward travelling wave excitation, }\end{cases}
$$

where $\mathbf{e}_{C+1}$ and $\mathbf{e}_{N-C+1}$ denote the $(C+1)$ th and $(N-C+$ 1)th columns of matrix $\mathbf{E}$. By substituting $\mathbf{f}(t)$ in equation
(18) into equation (14) and utilizing the orthogonality of each column of $\mathbf{E}, \widetilde{\mathbf{f}}(t)$ can be further expressed as

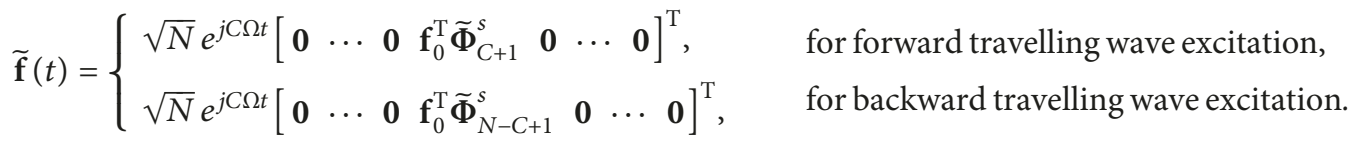

Finally, the modal coordinates $\boldsymbol{\eta}(t)$ can be obtained by equation (13), and the forced response of the mistuned system $\mathbf{u}(t)$ can be calculated via equation (6). Of course, the retained terms in equation (13) are determined according to the case of interest and should be consistent with those in equation (5).

\section{Model Validation}

In this section, the developed method is validated by comparing the results obtained by ROM with those by the full-order FE model. Figure 1 shows the FE model of a bladed disk, which consists of 17 blades. Quadratic tetrahedron elements are employed to discretize the structure and the resulting total number of DOFs is 304,266, where its sector model possesses 19,116 DOFs. The ROM has 170 DOFs with 10 modes retained per ND. The number of selected modes of cantilevered blade in mistuning projection equals 30. Such reduced-order model is employed in this and following sections for numerical simulation. The material of the structure is an alloy steel with Young's modulus $E=214.6 \mathrm{GPa}$, Poisson's ratio $v=0.3006$, and density $\rho=7850 \mathrm{~kg} / \mathrm{m}^{3}$. The Rayleigh damping model is used in this paper and $\mathbf{C}_{d}=\beta \mathbf{K}_{e}$, where $\beta$ denotes the damping coefficient and $\beta=1 \times 10^{-6}$ s.

Figure 2 shows the evolutions of natural frequencies of the tuned bladed disk versus the number of nodal diameter at the rotating speeds of $1000 \mathrm{rad} / \mathrm{s}$ and $2000 \mathrm{rad} / \mathrm{s}$, where the STA, SOF, and STF cases are included. As the Coriolis matrix lies in the damping term and is hardly contained in cyclic-symmetric analysis, the COR case is not considered. Although the last two cases are named as SOF and STF, the Coriolis matrix is not included during the analysis. The eigenvalues of the STA case are independent on rotating speed and are commonly used in the dynamic analysis of bladed disks to explore the sensitive frequency ranges of excitations. In contrast, the eigenvalues of the SOF and STF cases differ between different rotating speeds, as the spin softening and stress stiffening matrices are all speed-dependent. It can be seen in the figures that the spin softening and stress stiffening effects may lead to obvious influences in the eigenvalues, especially for high rotating speed. These results could reflect, in some extent, the influences of rotordynamic effects on the vibration of bladed disks.

Figure 3 displays the comparison of the evolution of natural frequencies versus rotating speed between the full-order model and ROM of the tuned bladed disk, where the Coriolis forces, spin softening, and stress stiffening effects are all taken into account. The stress stiffening matrix of the full-order model is obtained via statics analysis at different rotating speeds, and that of the ROM is acquired by the developed method shown in Appendix. It can be seen that the natural frequencies predicted by the ROM agree well with those by the fullorder model with the maximum relative error being less than $0.2 \%$. As can be seen in the figure, the natural frequencies at high rotating speed differ obviously from those at static condition. Almost all of the natural frequencies increase with the rotating speed, and some of the natural frequencies tend to split under the influence of the rotordynamic effects.

In order to validate the accuracy of the ROM in forced response analysis, comparison on the response of mistuned bladed disk is conducted. As the full-order model with quadratic elements is too large to analyse, its counterpart with linear tetrahedron elements, which has 49,266 DOFs, is employed. Correspondingly, the ROM is obtained by the sector model with linear elements of 3,273 DOFs. The variations in models exist only in the element type. The overall number of elements is not changed. Despite the different models employed in analysis, the comparison of the response can illustrate the effectiveness of the developed method. The proportional mistuning pattern of blade used during the analysis is listed in Table 1. The excitation is applied at the tip of each blade with the amplitude of $1 \mathrm{~N}$.

Figure 4 shows the comparison on the forced responses between the full-order model and ROM excited by a $4 \mathrm{EO}$ backward traveling wave excitation, where the four cases in equation (5) are all included. The speed ranges near the resonant peaks are of interest and depicted in figures. As the maximum response may exist in different blades for different rotating speeds, the envelope curves of the 


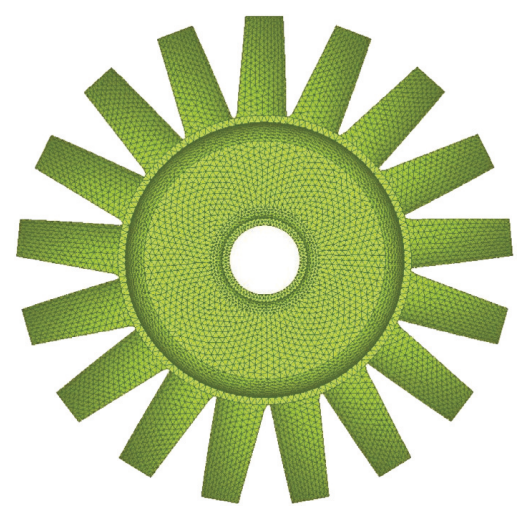

(a)

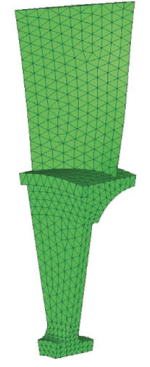

(b)

Figure 1: Finite element model of a bladed disk and its sector model.

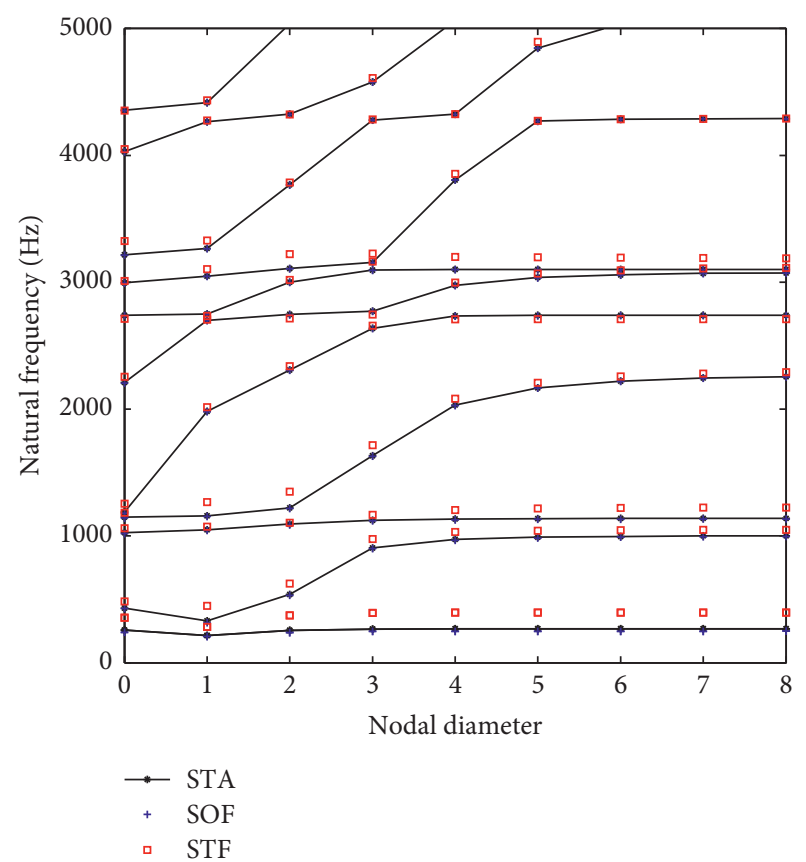

(a)

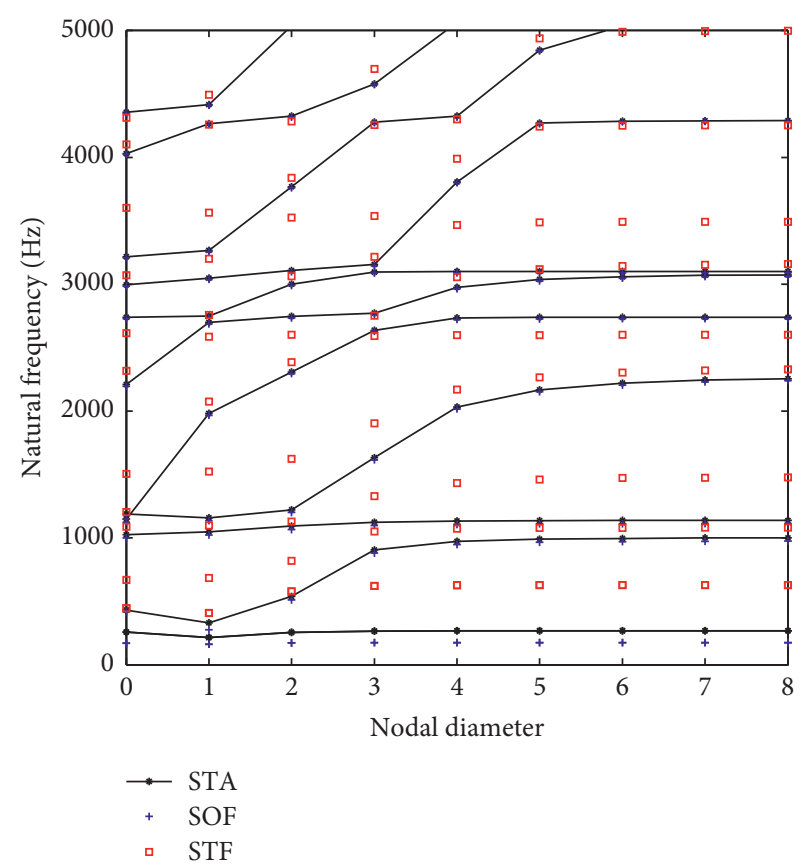

(b)

Figure 2: Natural frequencies versus the number of nodal diameters of the STA, SOF, and STF cases: (a) $\Omega=1000 \mathrm{rad} / \mathrm{s}$. (b) $\Omega=2000 \mathrm{rad} / \mathrm{s}$.

responses of all blades are used in this and following figures to more comprehensively present the vibration characteristics of mistuned bladed disks. It can be seen that the ROM gives very accurate responses in all four cases compared with the full-order model, where the maximum relative error in amplitudes is less than $0.5 \%$. Besides, the rotordynamic effects, especially the spin softening and stress stiffening effects, affect the forced response obviously in both the frequencies and the maximum amplitudes of resonant peaks. These effects will be discussed and explained detailedly in following sections. By the results shown in this section, the effectiveness of the developed method can be verified, which will be used to create ROM for the vibration analysis in following sections.

\section{Numerical Results}

4.1. Influences of Coriolis Forces, Spin Softening, and Stress Stiffening Effects on the Vibration of Rotating Mistuned Bladed Disks. In this section, the individual influences of Coriolis forces, spin softening, and stress stiffening effects on the forced responses of tuned and mistuned bladed disk are presented by considering these factors in sequence. First, the forced response of tuned and mistuned bladed disk versus rotating speed is depicted in Figures 5 and 6, where the STF case is included and the excitations are backward types.

As can be seen in Figures 5 and 6, the resonant peaks shift towards lower rotating speed as EO increases. This is easy to understand that the frequency of external force, as 


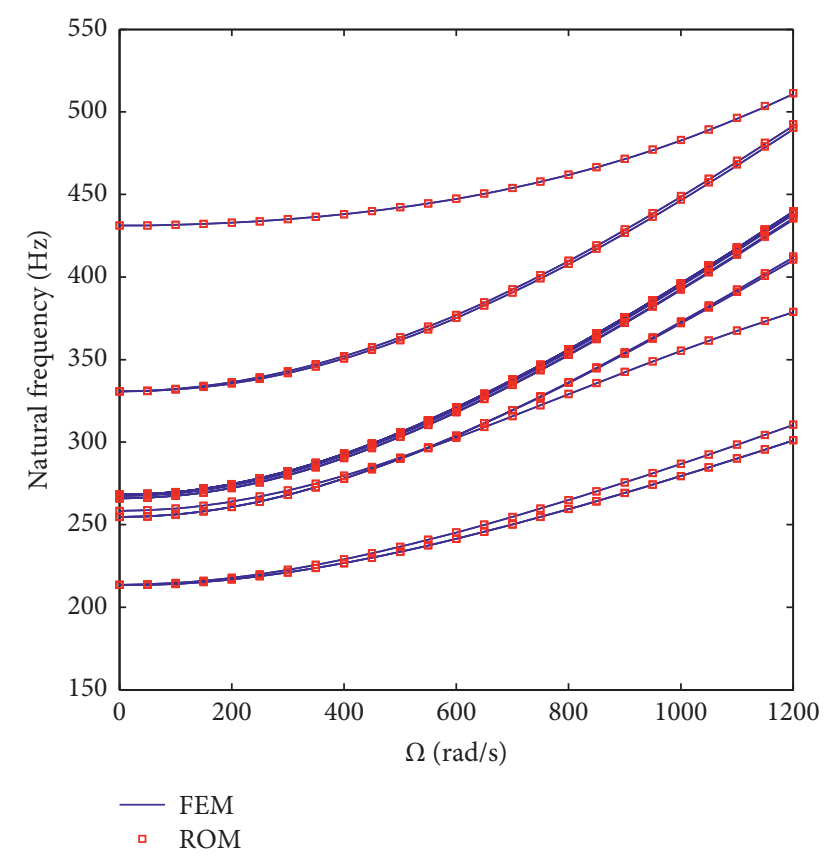

FIgURE 3: Evolution of first eight natural frequencies versus rotating speed of the full-order model and reduced-order model considering the Coriolis forces, spin softening, and stress stiffening effects.

TABLE 1: Mistuning parameters of bladed disk.

\begin{tabular}{lc}
\hline Blade & $\delta_{n}(\%)$ \\
\hline 1 & 0.00 \\
2 & 3.33 \\
3 & 0.47 \\
4 & -0.48 \\
5 & 1.90 \\
6 & 0.95 \\
7 & -2.86 \\
8 & -1.43 \\
9 & 3.33 \\
10 & 2.85 \\
11 & 1.42 \\
12 & -0.96 \\
13 & -3.80 \\
14 & 3.81 \\
15 & -3.34 \\
16 & -1.91 \\
17 & 2.38 \\
\hline
\end{tabular}

shown in equation (2), depends on the EO of excitation, so the excitation of higher EO has larger frequency. Besides, the excited modes in these speed ranges are mainly those of the first order of each ND, which have similar values of natural frequencies. As the Coriolis force, spin softening, and stress stiffening effects are all strongly dependent on the rotating speed, their effects tend to be more significant in the range of high speed, namely, for the cases with smaller EOs. These are also of interest in this paper.

Then, the effects of Coriolis force on the forced response of bladed disk are discussed. Figure 7 presents the forced response of tuned bladed disk with and without considering the Coriolis force, where both backward and forward excitations, which are represented by abbreviations of "BW" and "FW," have also been taken into account. It can be seen in Figure 7(a) that the response of STA cases with BW and FW excitations is perfectly coincided. As shown in the other three figures, Coriolis force leads to the shift of resonant frequencies, although the extent is relatively weak and different for different EOs of excitation. The response curves and the corresponding resonant frequencies of BW and FW excitations are no longer identical. Such phenomenon has also been found by Nikolic in [31]. This is easy to understand by referring to the knowledge in the field of rotordynamics that the Coriolis force tends to split the natural frequency pairs of identical values into backward ones and forward ones.

Figure 8 illustrates the comparison on the forced response of mistuned bladed disk with and without considering the Coriolis force, where the mistuning parameter is listed in Table 1. As can be seen in Figure 8, the consideration of Coriolis force leads to slight variation in the forced response. Figure 9 depicts the forced response of mistuned bladed disk excited by backward and forward traveling wave excitations. It can be seen that the direction of traveling wave has also visible influences on the forced responses of mistuned bladed disk, especially in the maximum amplitudes.

Regarding the influences of the spin softening and stress stiffening effects, Figure 10 shows the forced responses of the STA and SOF cases excited by backward traveling wave excitation of 2EO, and Figure 11 depicts those of the STA, SOF, and STF cases of 3EO excitation. Because the 2EO resonant frequencies of STF case are much larger than the STA and SOF cases, as is shown in Figures 5(a) and 6(a), its response is not included in Figure 10 for the case of clarity. It can be seen in the figures that the spin softening and stress stiffening effects, compared with the Coriolis force, have much more significant effects on the forced response of tuned and mistuned bladed disk, where the most obvious phenomenon is the shifts of the resonant peaks. The spin softening effect tends to decrease the resonant frequencies, whereas the stress stiffening effect plays the role of increasing the resonant frequencies. Besides, the degree of variation due to the latter one is much larger than that due to the former one. Moreover, the maximum response amplitude is also affected by these two effects. Just as the appellations of these two effects mean, the spin softening effect results in the reduction in the overall stiffness and decreases the resonant frequencies, whereas the stress stiffening effect contributes to the increase of stiffness.

The vibration localization phenomenon is another important issue in dynamic analysis of mistuned bladed disk. Figures 12 and 13 show the response amplitudes of blade tips of mistuned bladed disk excited by 3EO backward and forward traveling wave excitations, where the four cases are included. The exciting frequencies of each case are selected by picking the second resonant peak of the response curves of mistuned case. As depicted in the figures, the rotordynamic effects could affect the amplitude distribution of blades, where the effects of the stress stiffening effect are the 


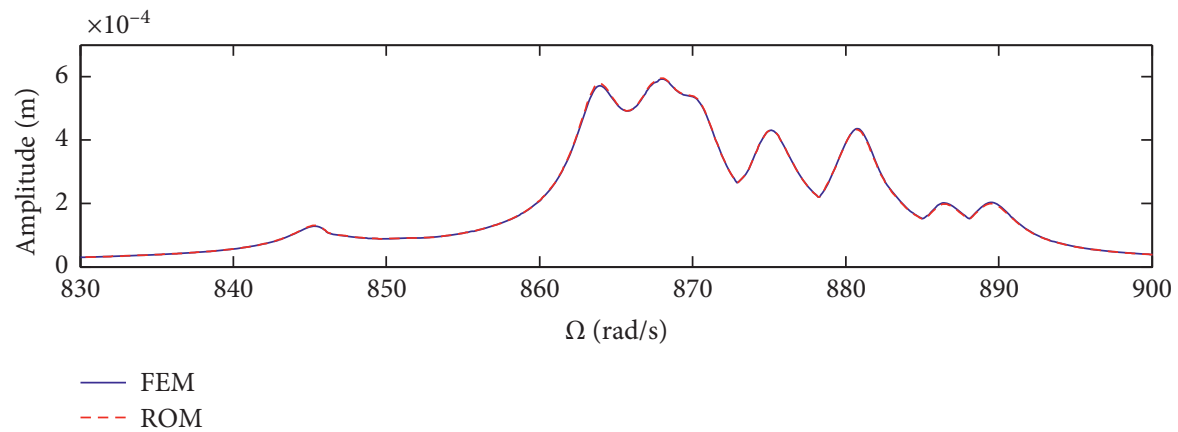

(a)

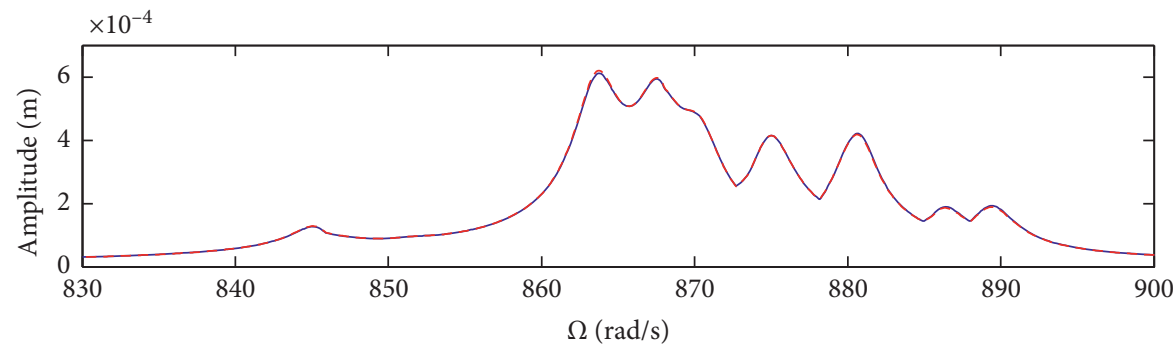

- FEM

- - ROM

(b)

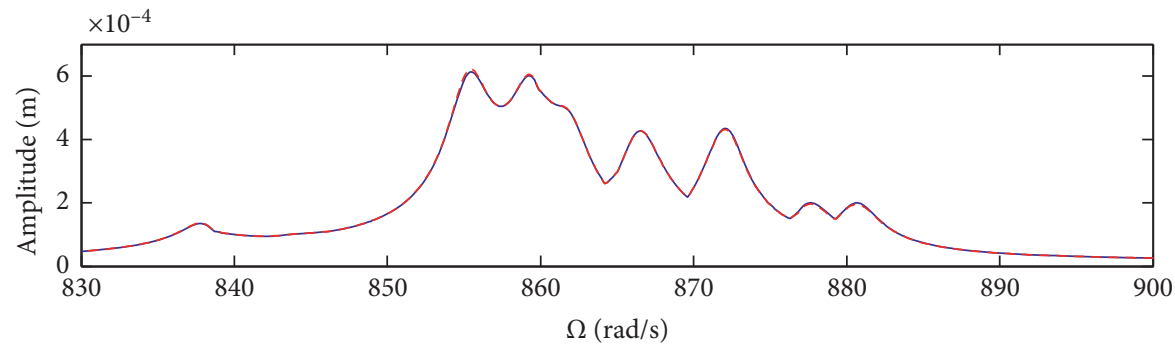

- FEM

--- ROM

(c)

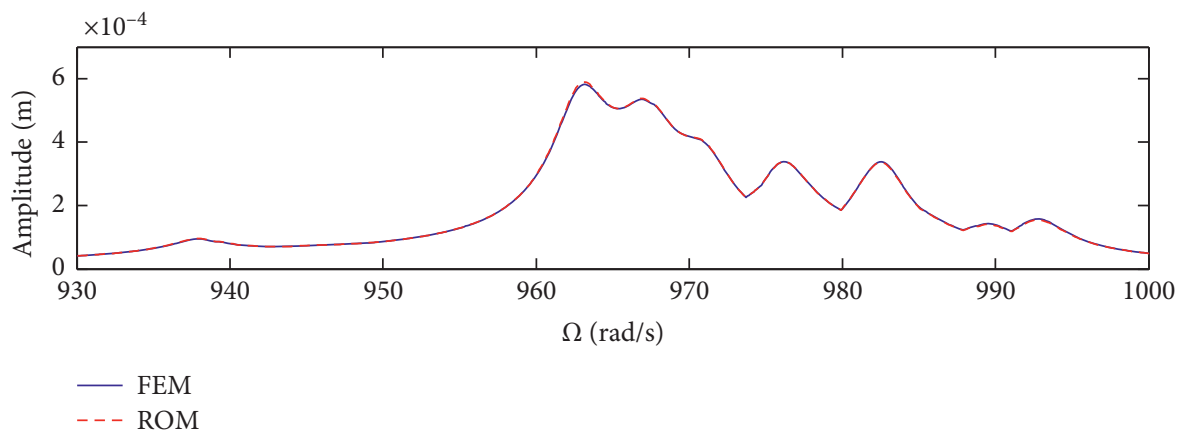

(d)

FIGURE 4: Comparison on the forced response versus rotating speed between the full-order model and ROM excited by a $4 \mathrm{EO}$ backward traveling wave excitation: (a) case STA, (b) case COR, (c) case SOF, and (d) case STF.

most significant. The direction of traveling wave excitations also affects the amplitude distribution between blades, as well as the maximum amplitude. For this particular occasion, the stress stiffening effect seems to obviously reduce the maximum response amplitudes, which may be due to the increase in overall stiffness.

Although a particular mistuning parameter is used in this section, it can be concluded by the results that the Coriolis force, 


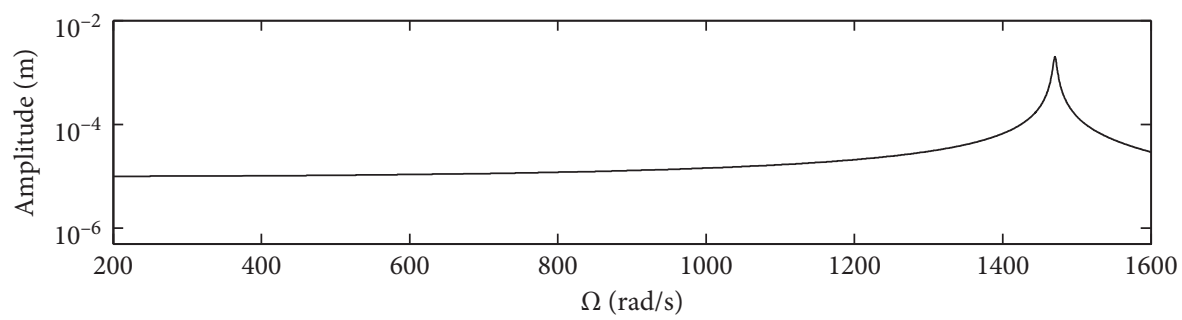

- STF: $2 \mathrm{EO}$

(a)

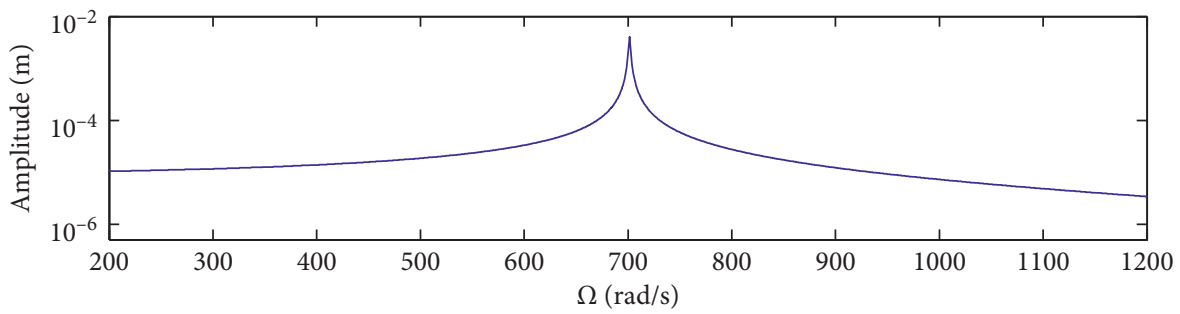

— STF: $3 \mathrm{EO}$

(b)

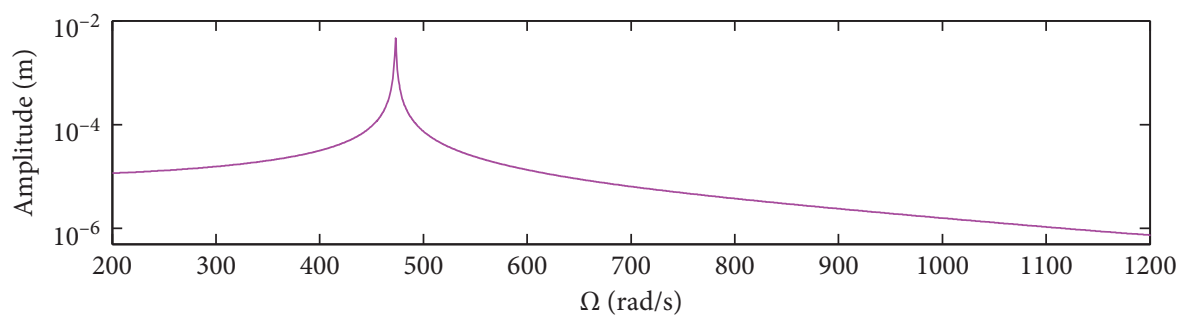

_ STF: $4 \mathrm{EO}$

(c)

FIGURE 5: Forced response of STF case of tuned bladed disk versus rotating speed excited by backward traveling wave excitations of (a) 2EO, (b) $3 \mathrm{EO}$, and (c) $4 \mathrm{EO}$.

spin softening, and stress stiffening effects can significantly affect the forced responses of mistuned bladed disks, especially in the shifts of resonant peaks. The response amplitudes also change to some extent due to these rotordynamic effects.

\subsection{Statistical Vibration Characteristics of Rotating Mistuned} Bladed Disks. In this section, the influences of Coriolis force, spin softening, and stress stiffening effects on the statistical characteristics of forced response of tuned and mistuned bladed disk are discussed. For the study on mistuning issue, employing only some particular mistuning cases is not enough to comprehensively reflect the vibration characteristics. Statistical analysis, by contrast, is a more suitable way for illustrating such issue. In this section, Monte Carlo simulation is employed to study the statistical characteristics of vibration of mistuned bladed disk considering the rotordynamic effects. Typically, amplitude magnification factor (AMF), which denotes the ratio of the largest response of blade in mistuned bladed disk to that in the tuned system, is used to quantitatively represent the response level of blade. Regarding the four cases discussed in this paper, eight quantitative AMFs are defined as

$$
\begin{aligned}
\mathrm{AMF}_{\mathrm{ABS}}^{\mathrm{STA}} & =\frac{u_{\mathrm{mist}}^{\mathrm{STA}}}{u_{\mathrm{tune}}^{\mathrm{STA}}}, \\
\mathrm{AMF}_{\mathrm{REL}}^{\mathrm{STA}} & =\mathrm{AMF}_{\mathrm{ABS}}^{\mathrm{STA}}, \\
\mathrm{AMF}_{\mathrm{ABS}}^{\mathrm{COR}} & =\frac{u_{\mathrm{mist}}^{\mathrm{COR}}}{u_{\mathrm{tune}}^{\mathrm{COR}}}, \\
\mathrm{AMF}_{\mathrm{REL}}^{\mathrm{COR}} & =\frac{u_{\mathrm{mist}}^{\mathrm{COR}}}{u_{\mathrm{tune}}^{\mathrm{STA}}}, \\
\mathrm{AMF}_{\mathrm{ABS}}^{\mathrm{SOF}} & =\frac{u_{\mathrm{mist}}^{\mathrm{SOF}}}{u_{\mathrm{tune}}^{\mathrm{SOF}}}, \\
\mathrm{AMF}_{\mathrm{REL}}^{\mathrm{SOF}} & =\frac{u_{\mathrm{mist}}^{\mathrm{SOF}}}{u_{\mathrm{tune}}^{\mathrm{STA}}}, \\
\mathrm{AMF}_{\mathrm{ABS}}^{\mathrm{STF}} & =\frac{u_{\mathrm{mist}}^{\mathrm{STF}}}{u_{\mathrm{tune}}^{\mathrm{STF}}}, \\
\mathrm{AMF}_{\mathrm{REL}}^{\mathrm{STF}} & =\frac{u_{\mathrm{mist}}^{\mathrm{STF}}}{u_{\mathrm{tune}}^{\mathrm{STA}}},
\end{aligned}
$$




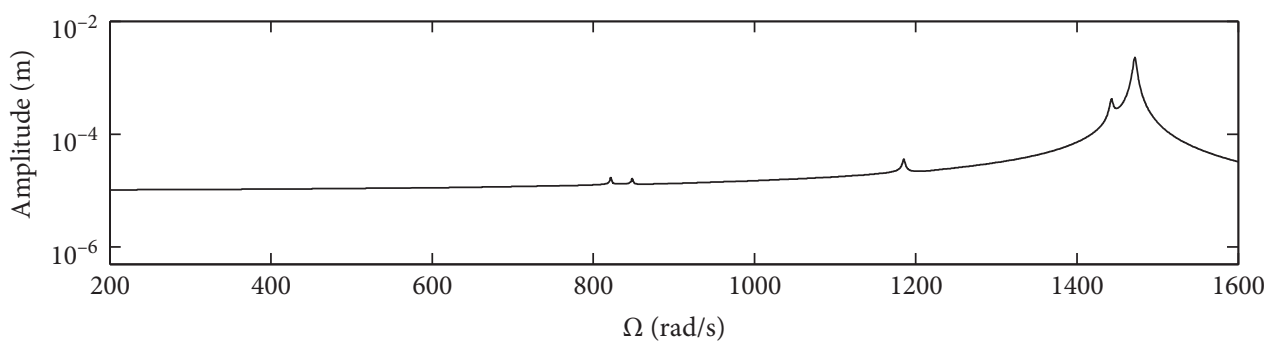

- STF: $2 \mathrm{EO}$

(a)

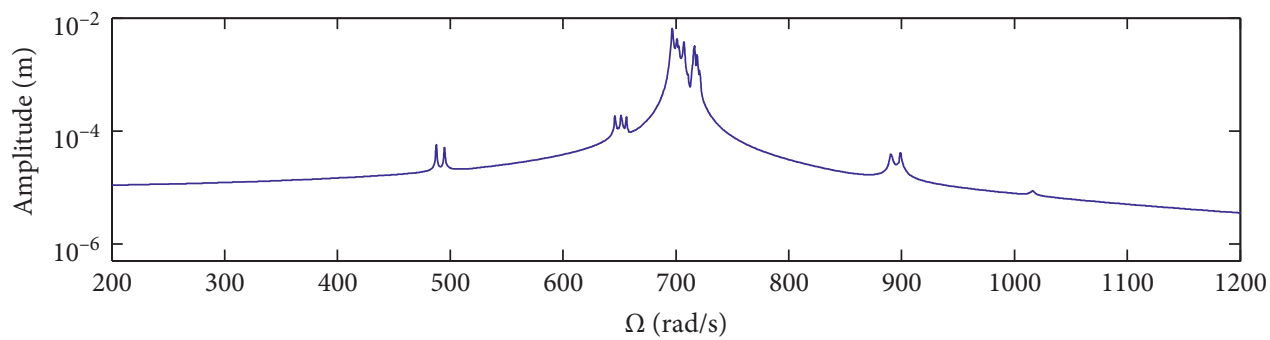

- STF: $3 \mathrm{EO}$

(b)

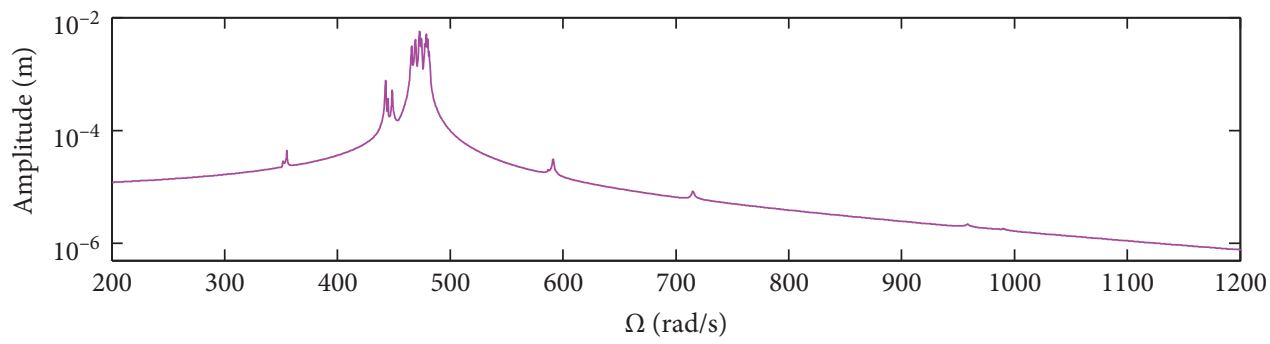

— STF: $4 \mathrm{EO}$

(c)

FIGURE 6: Forced response of case STF of mistuned bladed disk versus rotating speed excited by backward traveling wave excitations of (a) $2 \mathrm{EO}$, (b) $3 \mathrm{EO}$, and (c) $4 \mathrm{EO}$.
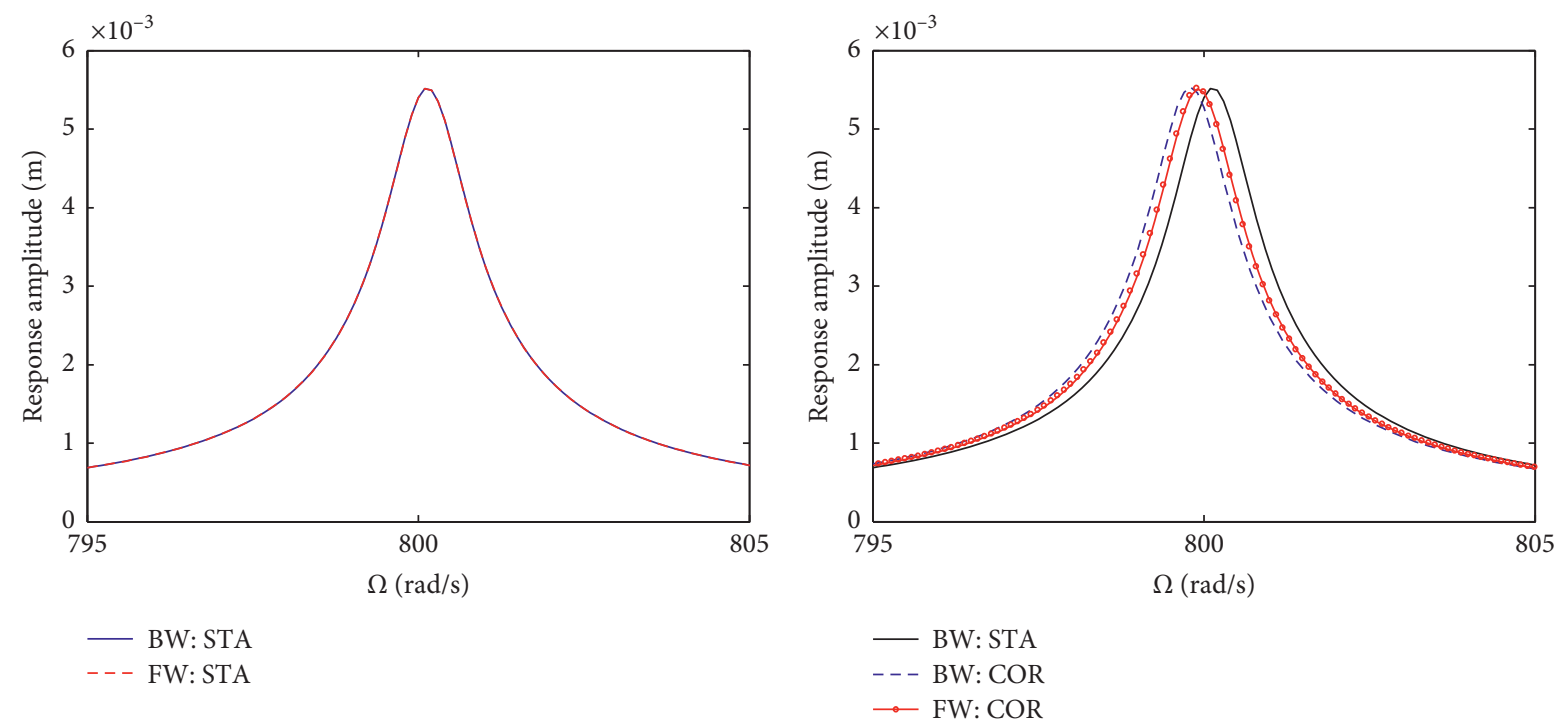

(a)

(b)

Figure 7: Continued. 


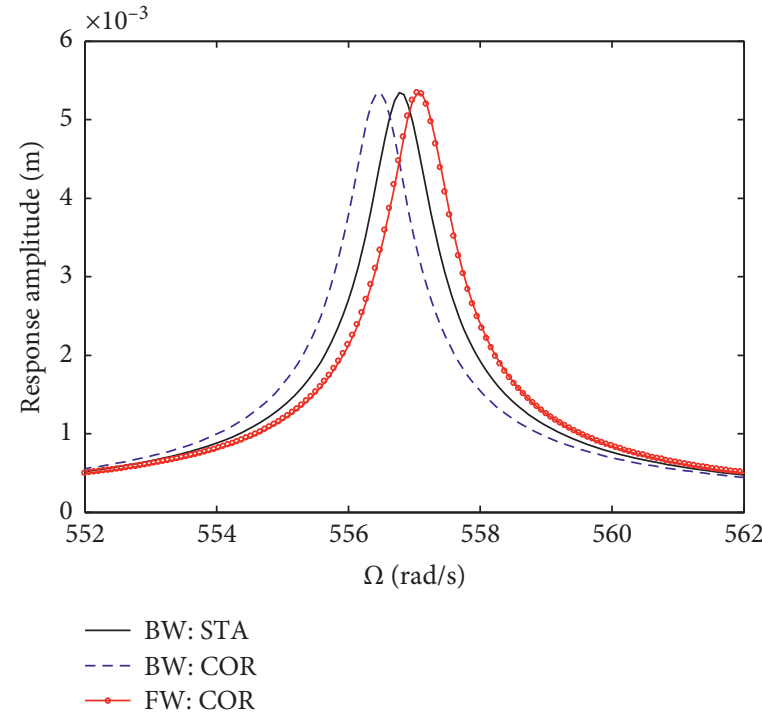

(c)

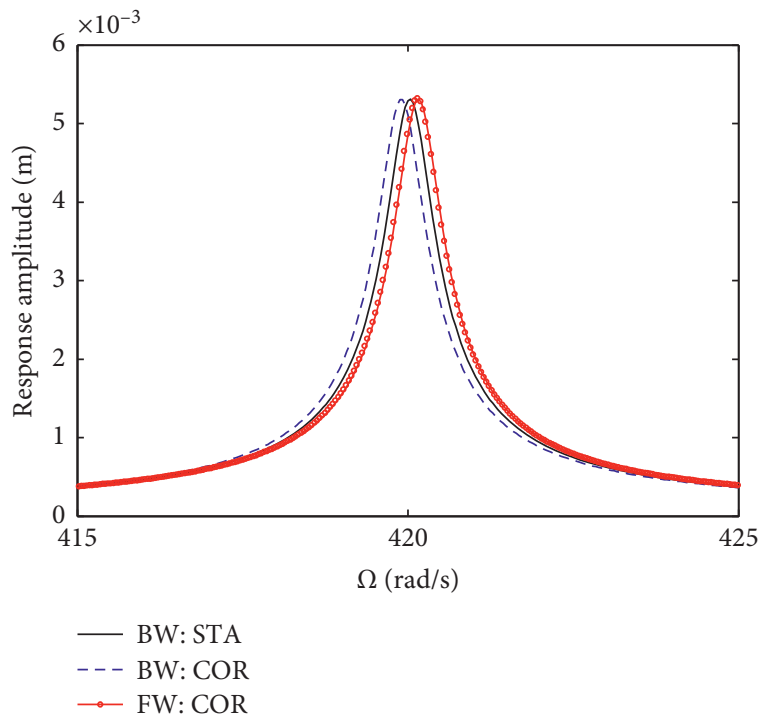

(d)

FIGURE 7: Forced response of tuned bladed disk with and without considering the Coriolis forces excited by backward and forward traveling wave excitations of (a) 2EO, (b) 2EO, (c) 3EO, and (d) 4EO.

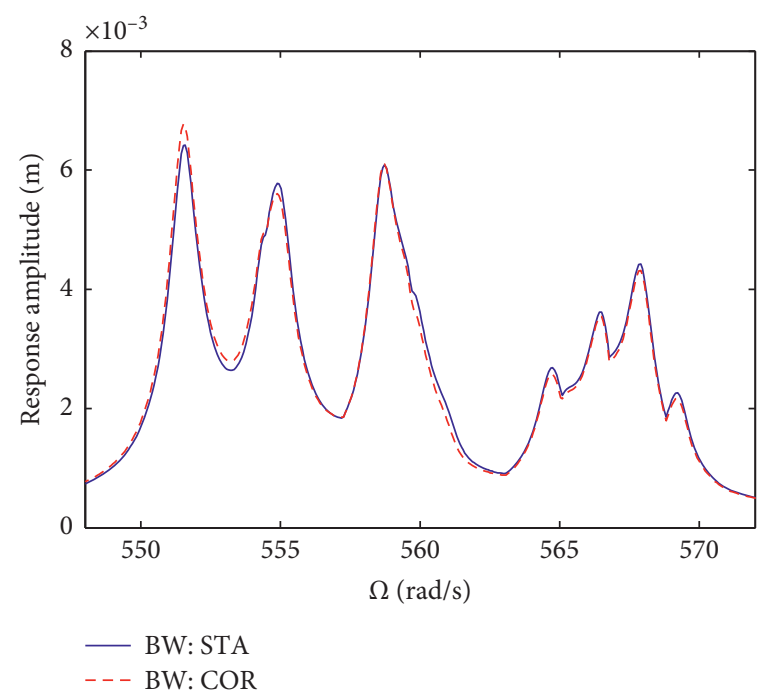

(a)

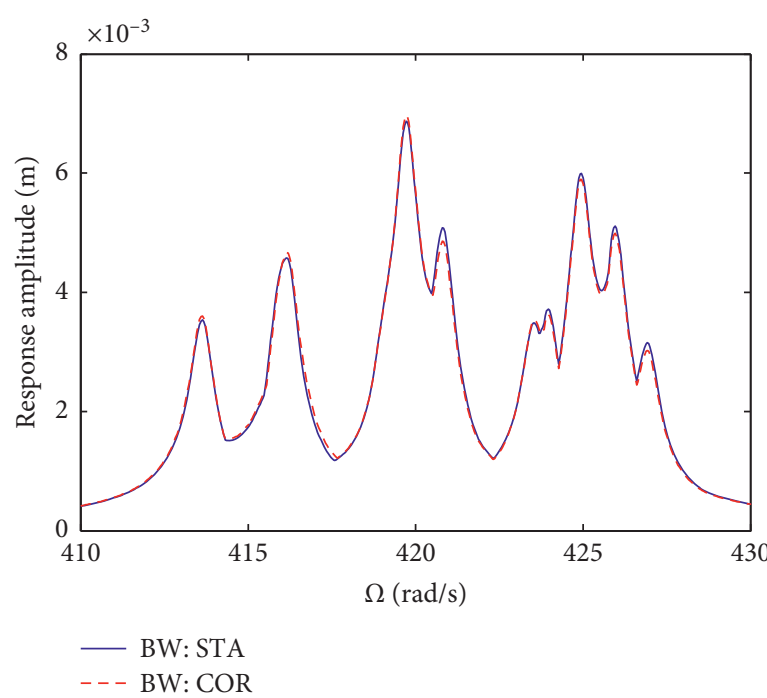

(b)

Figure 8: Forced response of mistuned bladed disk with and without considering the Coriolis forces excited by backward traveling wave excitations of (a) $3 \mathrm{EO}$ and (b) $4 \mathrm{EO}$.

where the subscript "ABS" and "REL" denote the absolute and relative AMFs, respectively. The variables in the denominators and numerators denote the maximum response amplitudes in the rotating speed range of interest. $\mathrm{AMF}_{\mathrm{REL}}^{\mathrm{STA}}$ is included in equation (20) for ease of description in following parts.

In this paper, the absolute and relative AMFs are defined because there are two reference values of the tuned system for the COR, SOF, and STF cases, such as $u_{\text {tune }}^{\mathrm{STA}}$ and $u_{\text {tune }}^{\mathrm{COR}}$ for case COR. Generally, these two reference values are not identical due to the influences of the rotordynamic effects, as are shown in Section 4.1. According to the definitions of these two kinds of factors, the relative ones could reflect the influences of rotordynamic effects on the statistical characteristics of forced response, by comparing with those of STA case. The absolute ones may give information about the realistic amplitude magnification caused by mistuning in operating conditions. Thus, these two kinds of AMFs could provide insights into mistuning via two different perspectives.

As stated in several investigations [20, 22, 34], the probability distributions of response amplitude of static mistuned bladed disks, namely, the STA case, conform to Weibull distribution. With such statistical model, the Monte 


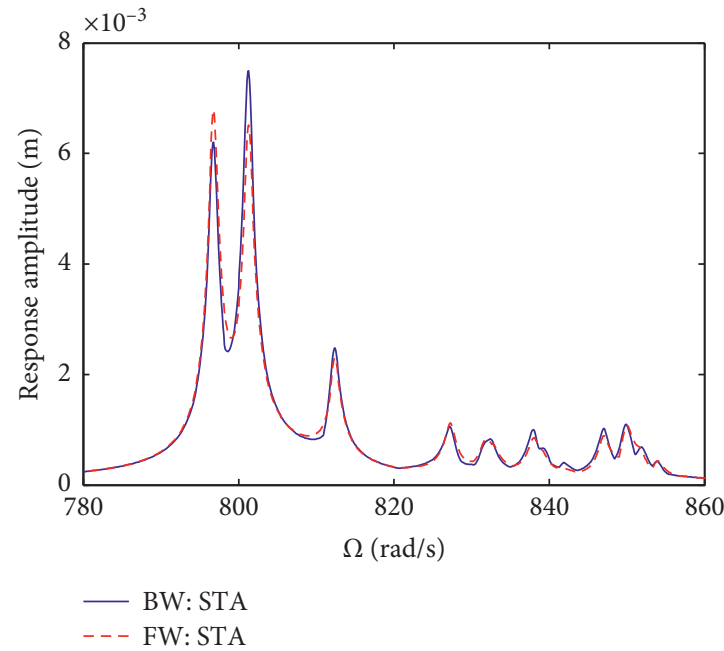

(a)

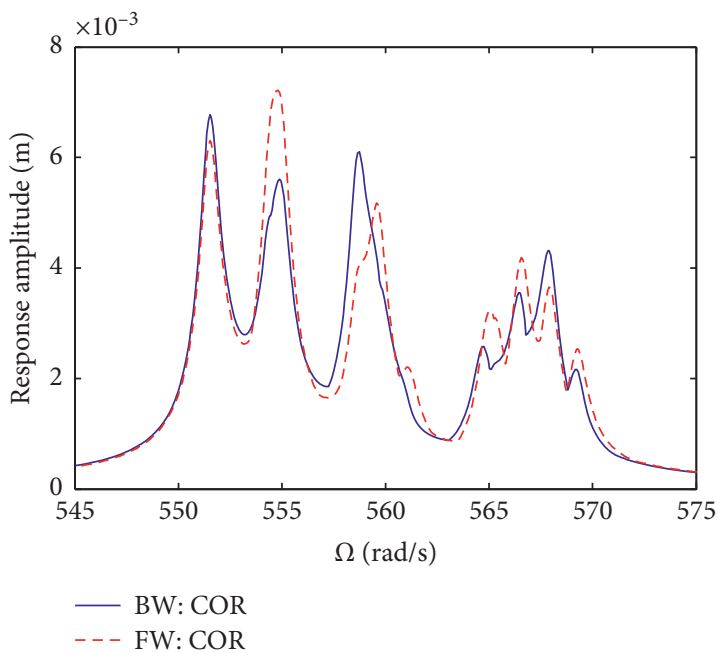

(b)

FIGURE 9: Forced response of mistuned bladed disk excited by backward and forward traveling wave excitations of (a) 2EO and (b) 3EO.

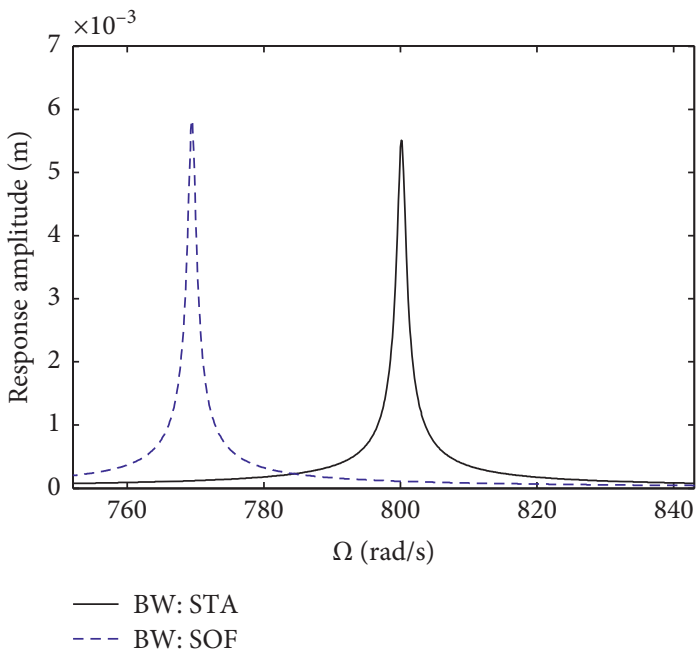

(a)

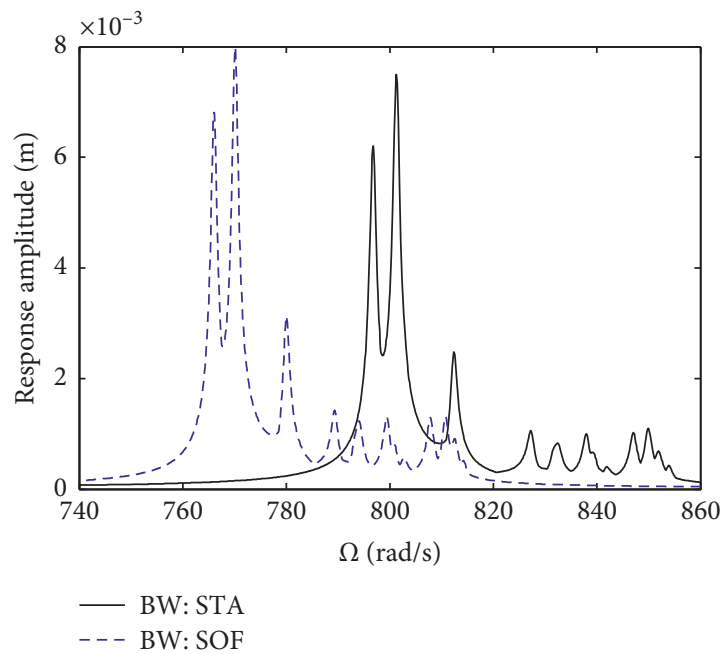

(b)

FIGURE 10: Forced response of tuned and mistuned bladed disk excited by backward traveling wave excitations of 2EO: (a) tuned case and (b) mistuned case.

Carlo simulation can be greatly accelerated, as relatively few mistuning patterns are needed to get the distribution parameters. Figure 14 shows the probability densities obtained by Monte Carlo simulation of 10000 mistuning patterns and by Weibull distribution via least square fitting. During the analysis, the mistuning parameter is generated by uniform distribution of mean zero and standard deviation $2.0 \%$. It can be seen in the figures that the probability densities versus absolute AMFs of COR, SOF, and STF cases are also conforming to Weibull distribution. Then, it can also be concluded that the probability densities of relative AMFs conform to Weibull distribution either, as $u_{\text {tune }}^{\mathrm{STA}}, u_{\text {tune }}^{\mathrm{COR}}, u_{\text {tune }}^{\mathrm{SOF}}$, and $u_{\text {tune }}^{\text {STF }}$ are all scalar and independent on the mistuning patterns. In following parts, the results of Weibull distribution fitted by 2000 random mistuning patterns are employed.
Figure 15 depicts the comparison on the probability density curves versus relative AMF under the action of backward traveling wave excitations of six different EOs. As the $2 \mathrm{EO}$ resonant frequency of STF case is much larger than those of the other three cases and is beyond the speed range of interest, its probability density curve is not included in Figure 15(a). According to definitions in equation (20), the relative AMF could reflect the variation in forced response of mistuned bladed disk with and without considering the rotordynamic effects. It can be seen in Figure 15 that the rotordynamic effects lead to obviously changes in the probability distributions of AMF, especially the stress stiffening effect. Moreover, their influences present different characteristics for different EOs. Detailedly, the influences of Coriolis forces are relatively weak for most cases except 3EO, and those of spin softening effect are 


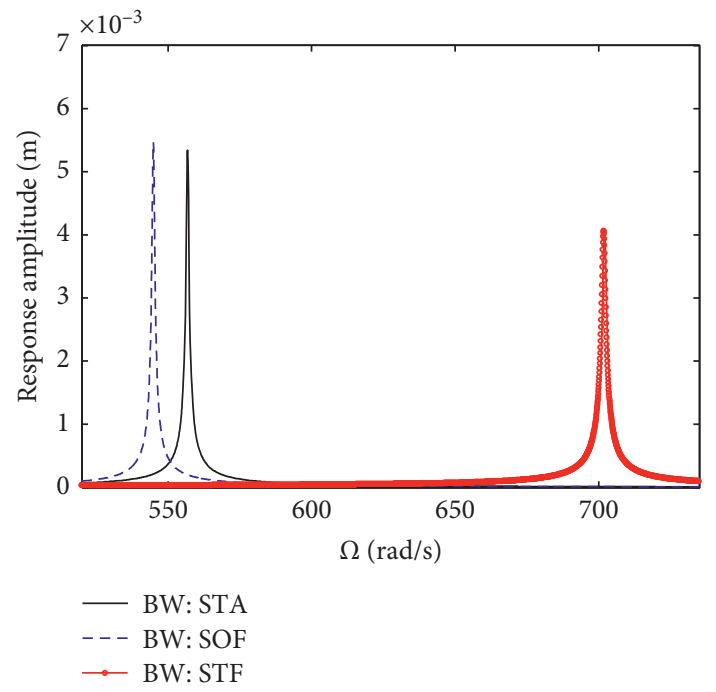

(a)

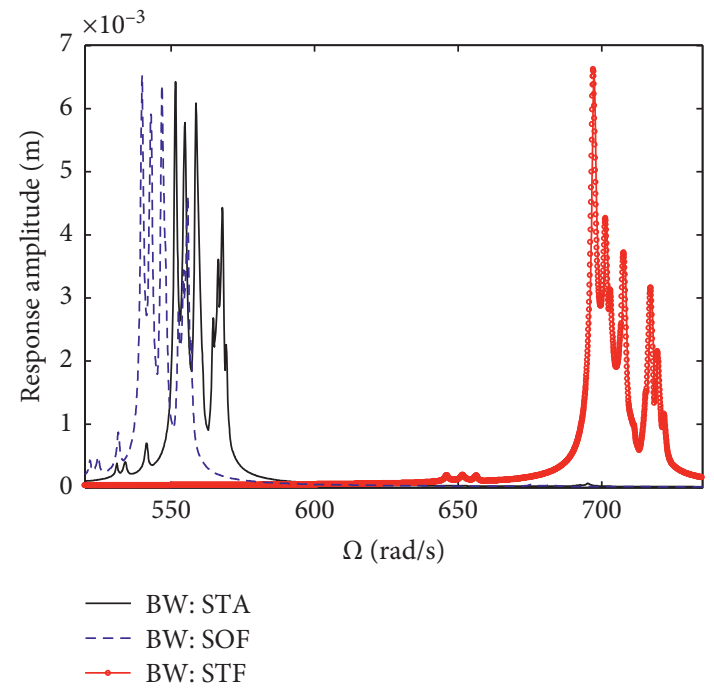

(b)

FIGURE 11: Forced response of tuned and mistuned bladed disk excited by backward traveling wave excitations of 3EO: (a) tuned case and (b) mistuned case.

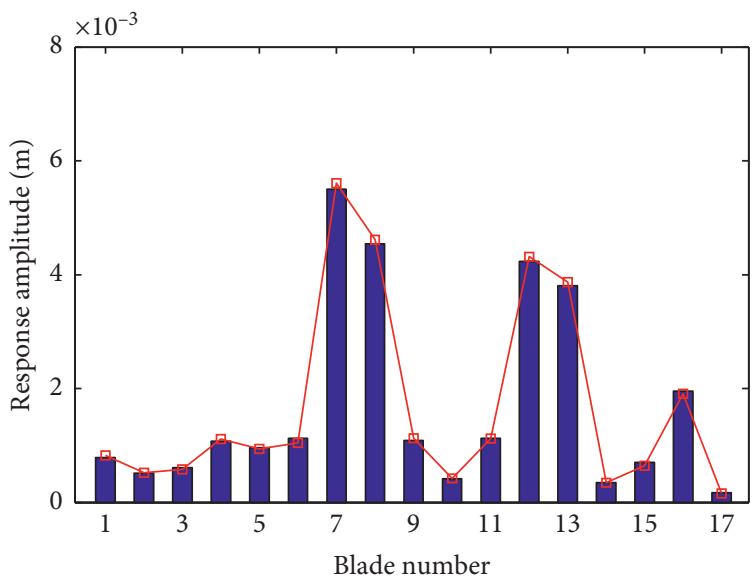

BW: $\mathrm{COR}$ $\rightarrow$ BW: STA

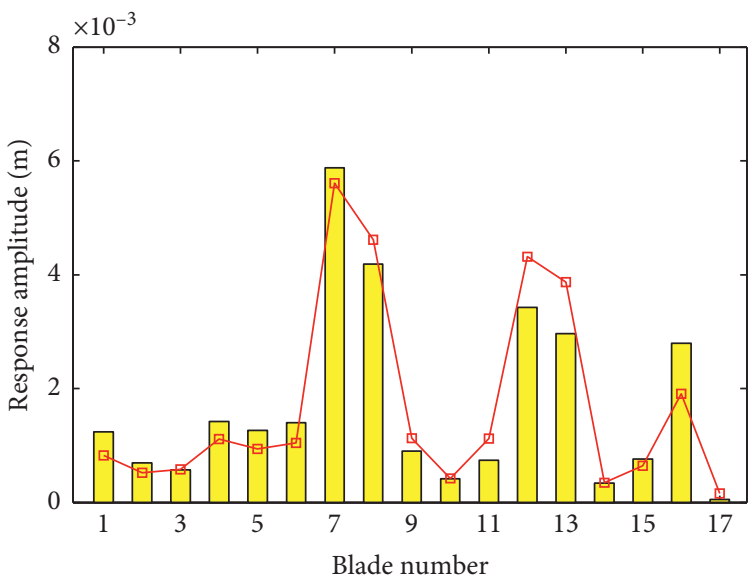

$\square$ BW: $\mathrm{SOF}$ $\rightarrow$ BW: STA

(b)

(a)

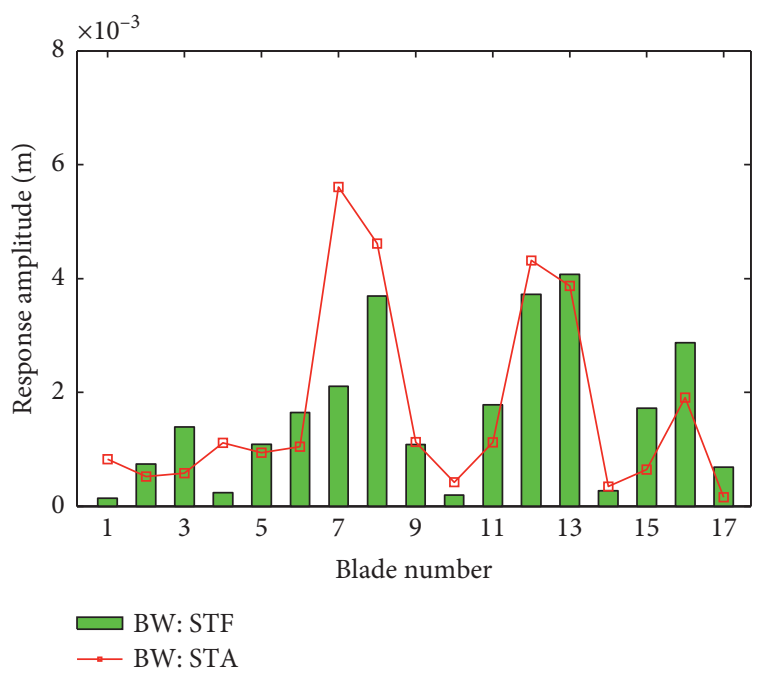

(c)

FIGURE 12: Response amplitudes of blade tips of mistuned bladed disk excited by a 3EO backward traveling wave excitation: (a) case COR, (b) case SOF, and (c) case STF. 


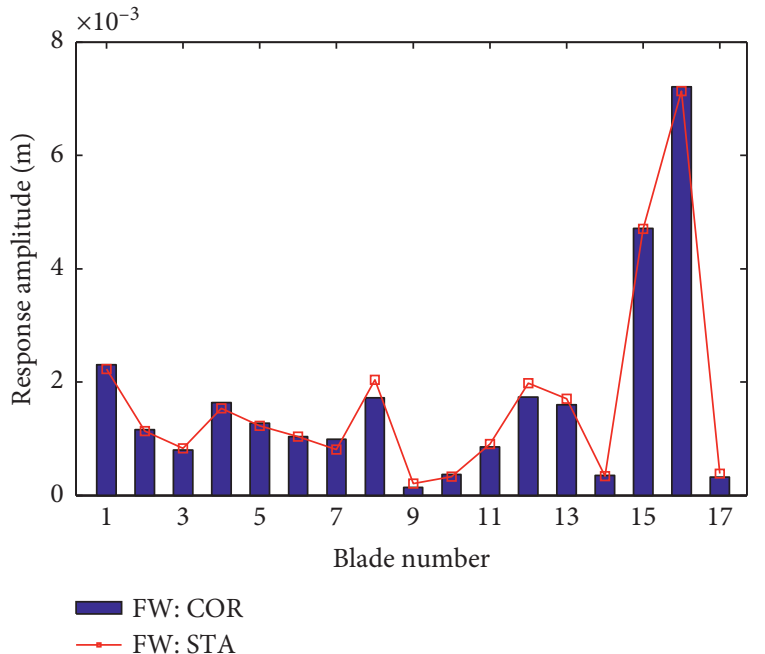

(a)

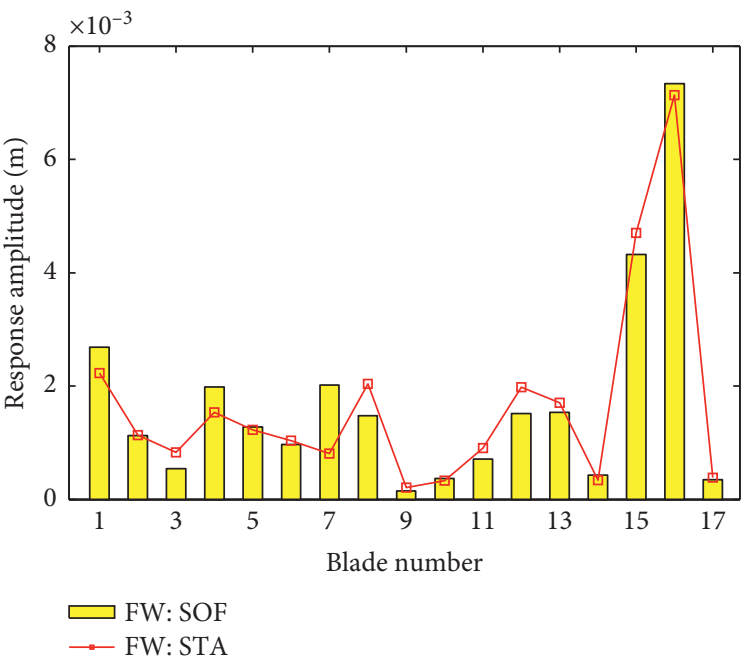

(b)

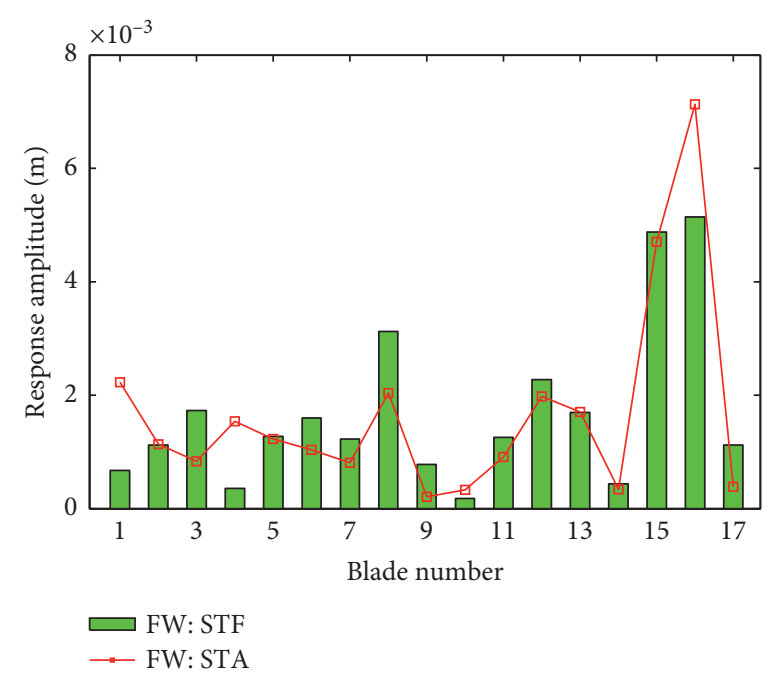

(c)

FIGURE 13: Response amplitudes of blade tips of mistuned bladed disk excited by a 3EO forward traveling wave excitation: (a) case COR, (b) case SOF, and (c) case STF.

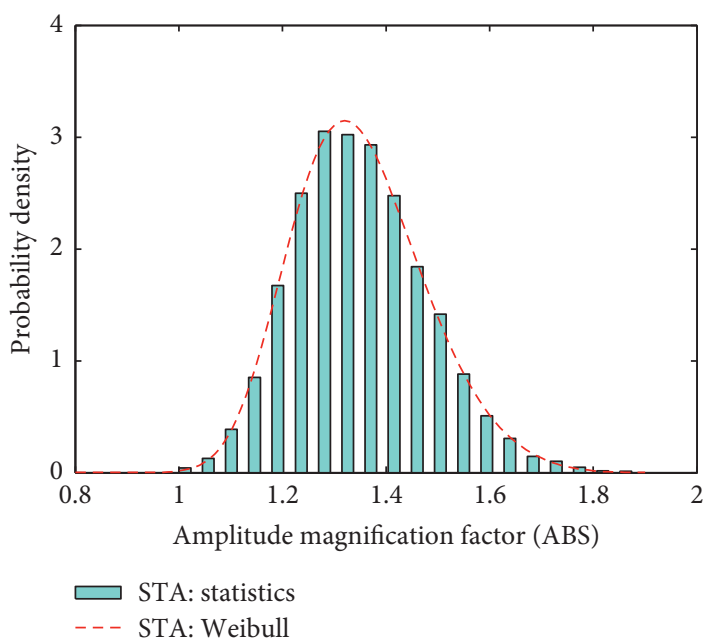

(a)

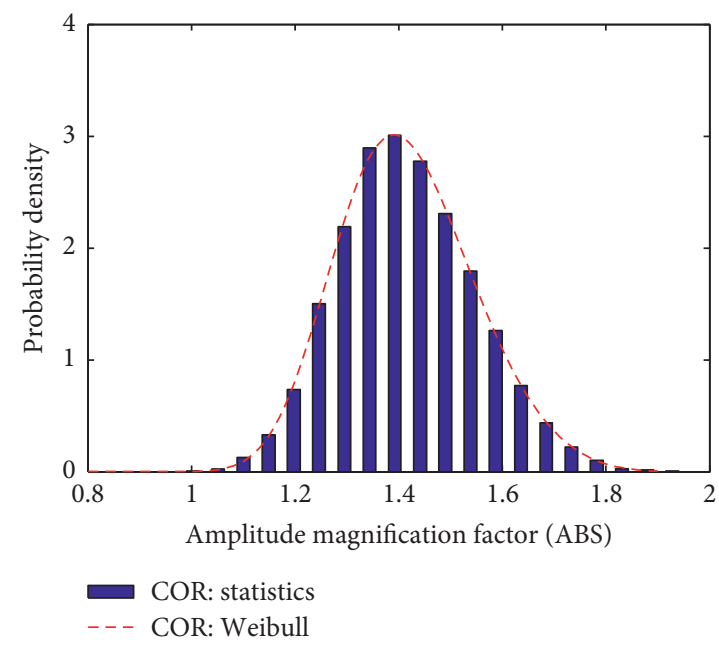

(b)

Figure 14: Continued. 


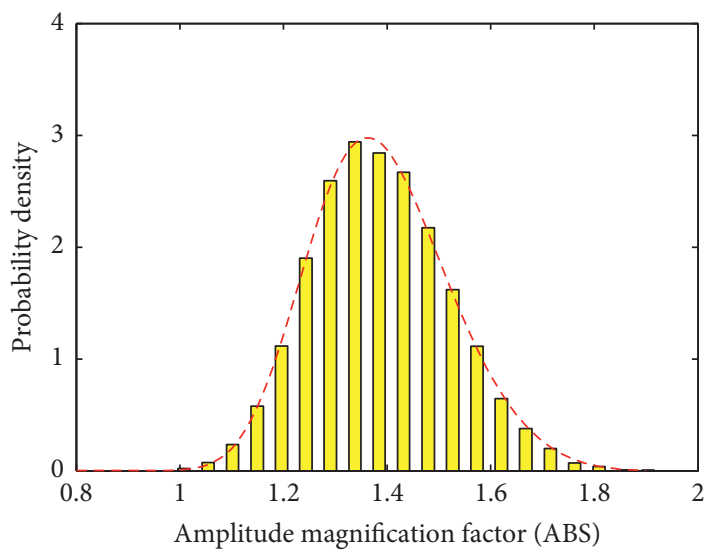

$\square$ SOF: statistics - - - SOF: Weibull

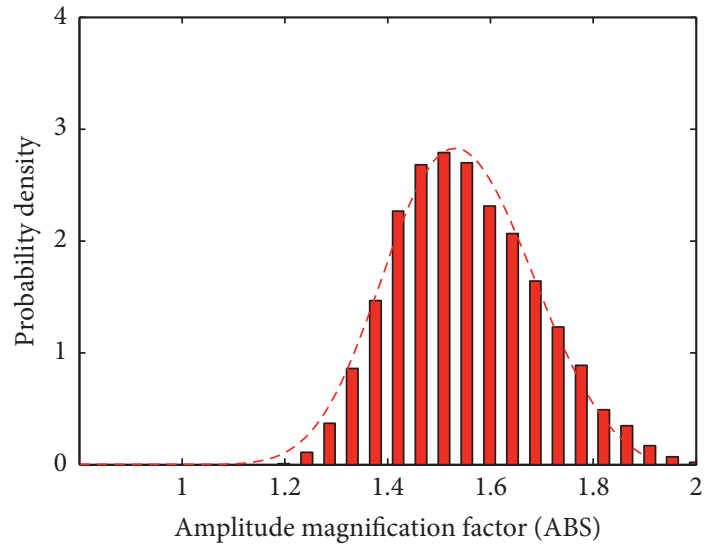

STF: statistics - - - STF: Weibull

(c)

(d)

FIGURe 14: Probability densities versus absolute AMF obtained by Monte Carlo simulation of 10000 mistuning patterns generated by uniform distribution of mean 0 and standard deviation 2.0\%: (a) case STA, (b) case COR, (c) case SOF, and (d) case STF.
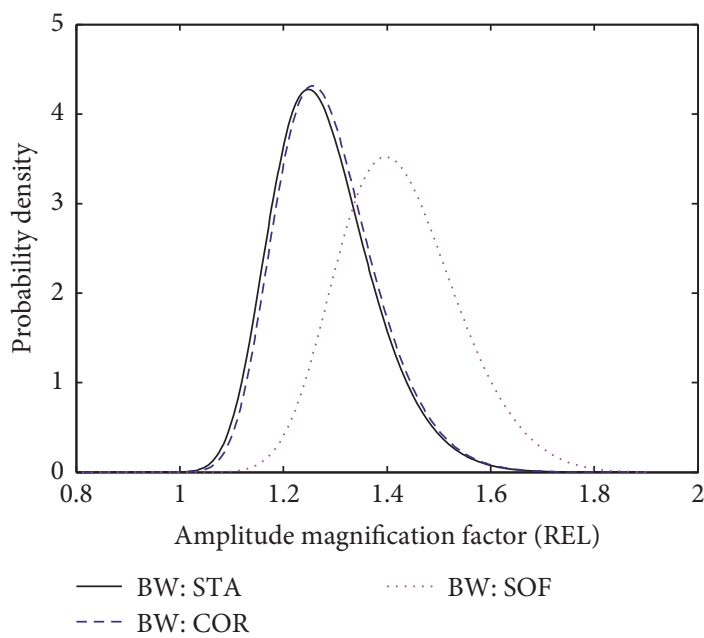

(a)

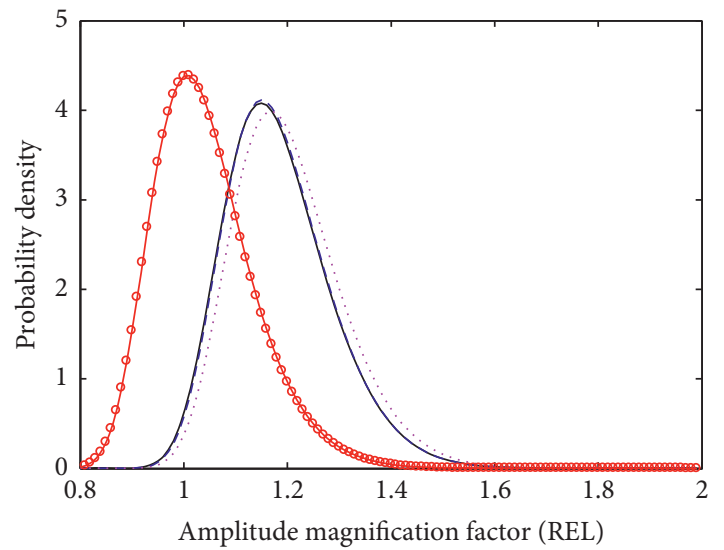

- BW: STA
-- BW: COR

…. BW: SOF

(c)

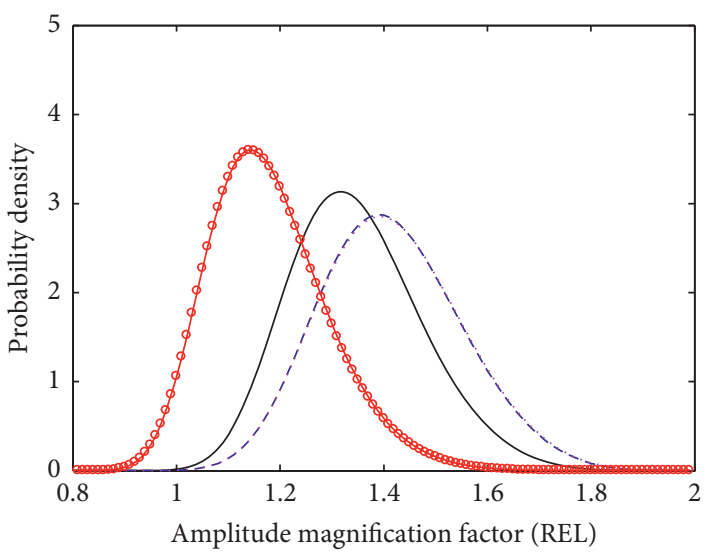

$\begin{array}{ll}- & \text { BW: STA } \\ --- & \text { BW: COR }\end{array}$

BW: SOF

$\rightarrow$ BW: STF

(b)

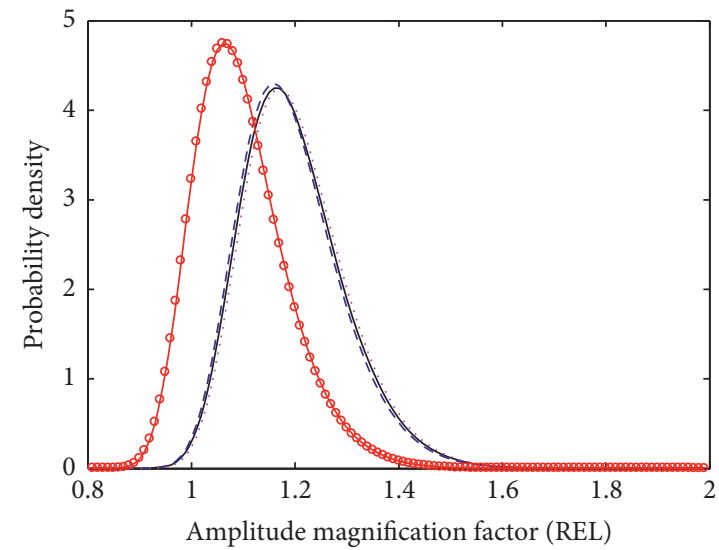

- BW: STA
-- BW: COR

BW: SOF

$\rightarrow$ BW: STF

(d)

Figure 15: Continued. 


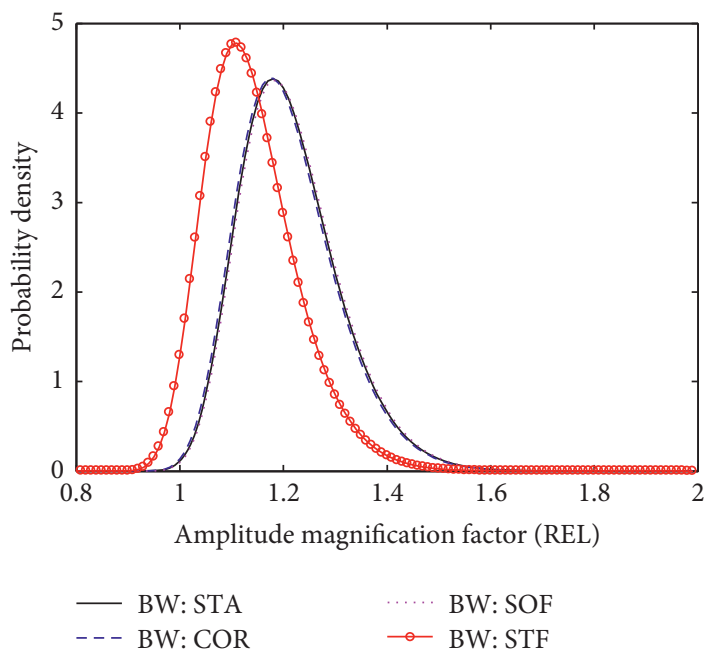

(e)

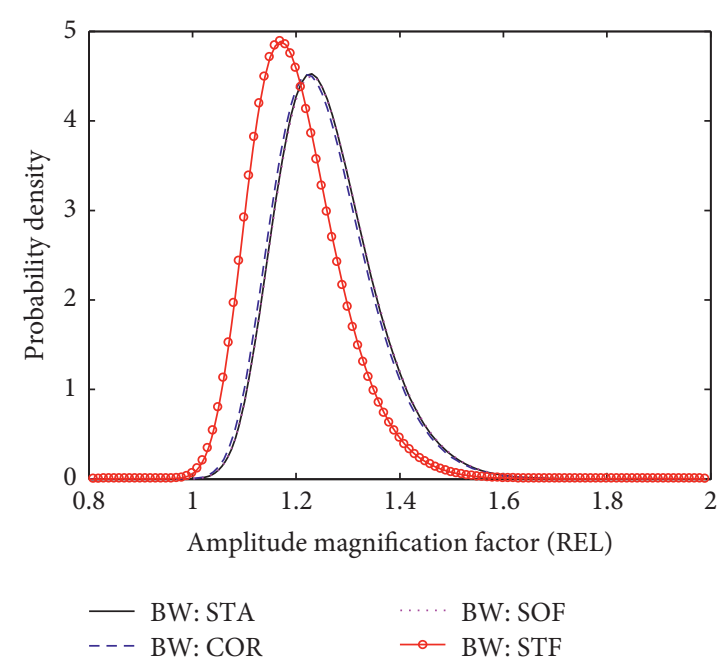

(f)

Figure 15: Probability densities versus relative AMF obtained by Monte Carlo simulation of 2000 mistuning patterns generated by uniform distribution of mean 0 and standard deviation $2.0 \%$ under the action of (a) 2EO, (b) 3EO, (c) 4EO, (d) 5EO, (e) 6EO, and (f) 7EO backward traveling wave excitations.

quite significant for $2 \mathrm{EO}$ and $3 \mathrm{EO}$. The stress stiffening effect plays an obvious role in all of the cases. Nevertheless, the extent of variation in probability distribution gradually decreases as EO increases.

Besides, compared with the probability density curves of STA case, the spin softening effect tends to shift the curves towards higher values of AMF, whereas the stress stiffening effect makes the AMF distribute within the range of smaller values. This phenomenon is consistent with the results shown in Section 4.1, which are all due to that the spin softening effect decreases the overall stiffness and the stress stiffening effect enhances the stiffness of the system. By the results shown in Figure 15, a common phenomenon can be seen that the influences of the rotordynamic effects are much more remarkable for the cases with lower EO, such as those shown in Figures 15(a) and 15(b). This can be interpreted by the fact that the resonant peaks of lower EO cases tend to possess higher rotating speeds, and thus, the rotordynamic effects are much more significant within these speed ranges.

For comparison, Figure 16 depicts the probability density curves versus absolute AMF. According to definitions in equation (20), the absolute AMF could reflect the realistic amplitude magnification due to mistuning in rotating conditions, as it is calculated by the ratio of maximum response amplitude of a certain mistuned case to that of the corresponding tuned case, where both cases consider the rotordynamic effects. As shown in Figure 16, the rotordynamic effects cause obvious changes in the probability distribution of absolute AMF for the cases of 2EO and $3 \mathrm{EO}$, whereas their influences on the $4 \mathrm{EO}$ and $5 \mathrm{EO}$ cases are negligible. Besides, Figures 16(a) and 16(b) also illustrate that the rotordynamic effects make the probability distribution shift towards higher values, where the stress stiffening effect plays the most significant role. This phenomenon indicates that the largest AMF of mistuned bladed disks that might encounter in rotating conditions may be much larger than that predicted by the models in static states.

Figures 15 and 16 depict only the results with respect to a particular standard deviation $2 \%$ of mistuning. In order to gain more comprehensive insights into the characteristics of AMF at different mistuning levels, Figure 17 shows the variation of $95^{\text {th }}$ percentile of relative AMF versus standard deviation of mistuning parameter. As can be seen in s, the variation curves all present the tendency of increasing first and then decreasing, where the maximum values exist at certain points with smaller standard deviation. This phenomenon has been found in previous investigations $[18,20,22]$. With respect to the specific influences of rotordynamic effects, the influences of rotordynamic effects on relative AMF are consistent with those shown in Figure 15. The Coriolis force and spin softening effect lead to slight changes in the curves, and the differences are negligible for small deviation cases. By contrast, the stress stiffening effect results in remarkable influences on the $95^{\text {th }}$ percentile of relative AMF over the entire range of standard deviation. With the conclusions drawn by Figure 15, such phenomenon is easy to understand and can be accounted for by the explanations in previous parts.

Figure 18 depicts the variation of $95^{\text {th }}$ percentile of absolute AMF versus standard deviation of mistuning parameter. It can be seen in Figure 18 that the influences of Coriolis force and spin softening effect are still relative weak and are negligible for some cases. The stress stiffening effect leads to obvious variation in the curves, especially for the $3 \mathrm{EO}$ case. The most significant changes are the increase of AMF in the range of $(2 \%, 6 \%)$ and the shift of the point with maximum value towards higher value. Nevertheless, for the cases with relatively small mistuning, the absolute AMF exists to be smaller than that at static state. 

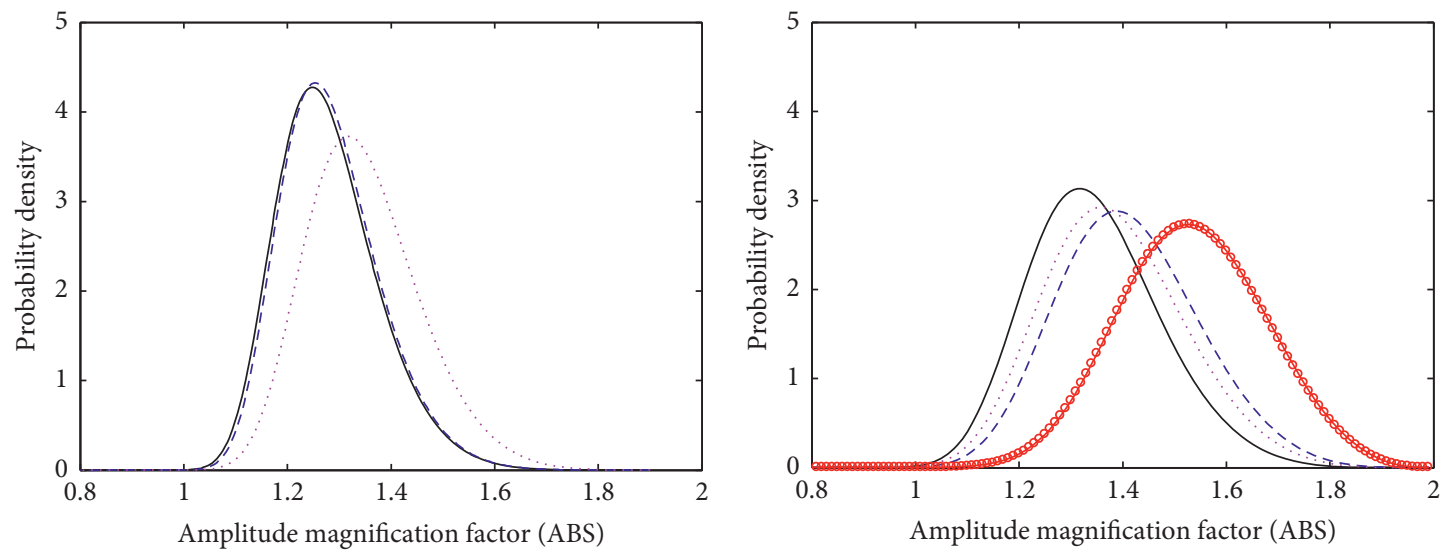

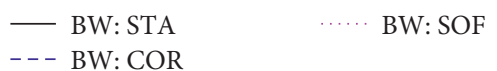

(a)

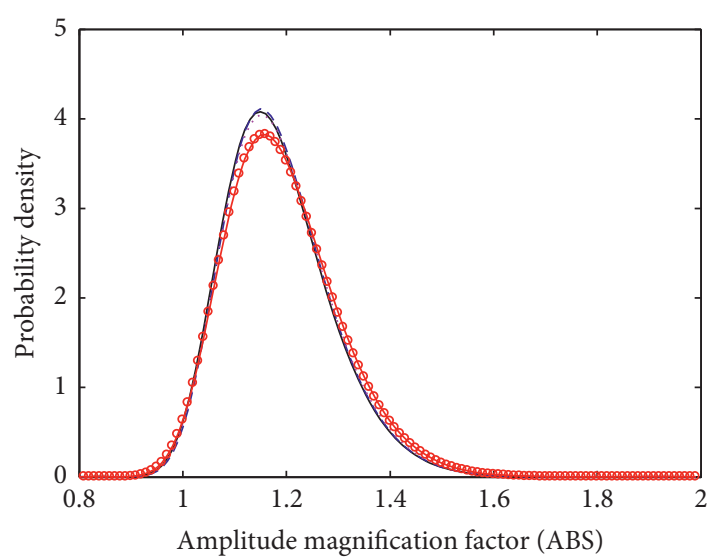

Amplitude magnification factor (ABS)

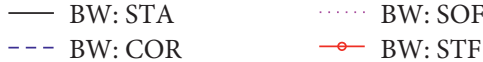

(c)

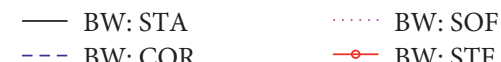

(b)

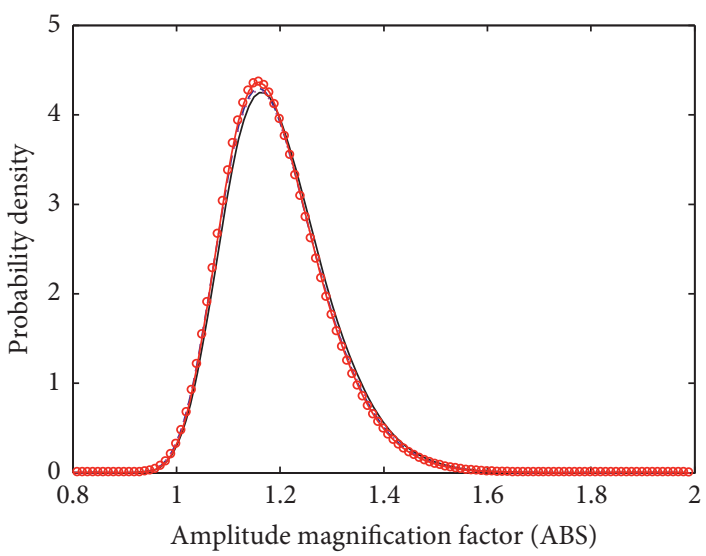

(d)

FIgure 16: Probability densities versus absolute AMF obtained by Monte Carlo simulation of 2000 mistuning patterns generated by uniform distribution of mean 0 and standard deviation $2.0 \%$ under the action of (a) 2EO, (b) 3EO, (c) 4EO, and (d) 5EO backward traveling wave excitations.

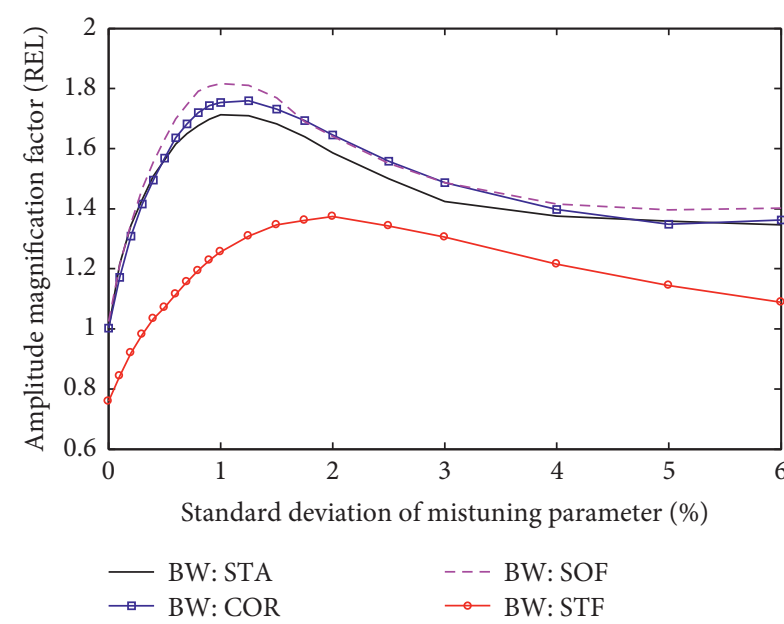

(a)

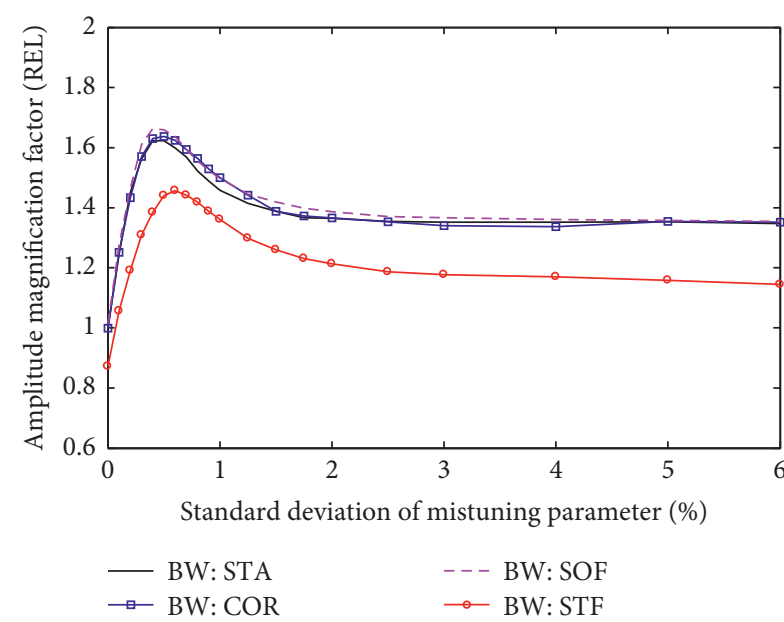

(b)

FIGURE 17: $95^{\text {th }}$ percentile of relative AMF versus standard deviation of mistuning parameter under the action of (a) $3 \mathrm{EO}$ and (b) $4 \mathrm{EO}$ backward traveling wave excitations. Monte Carlo simulation of 2000 mistuning patterns generated by uniform distribution of mean 0 is conducted for each point. 


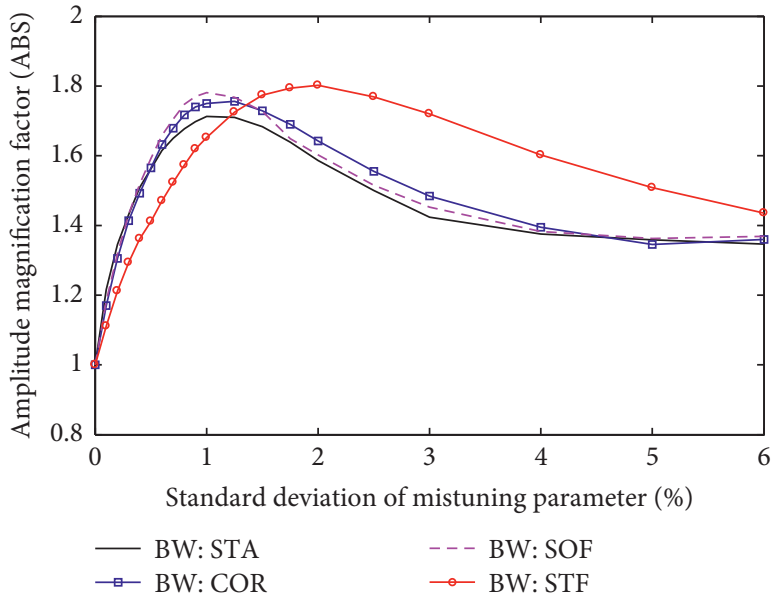

(a)

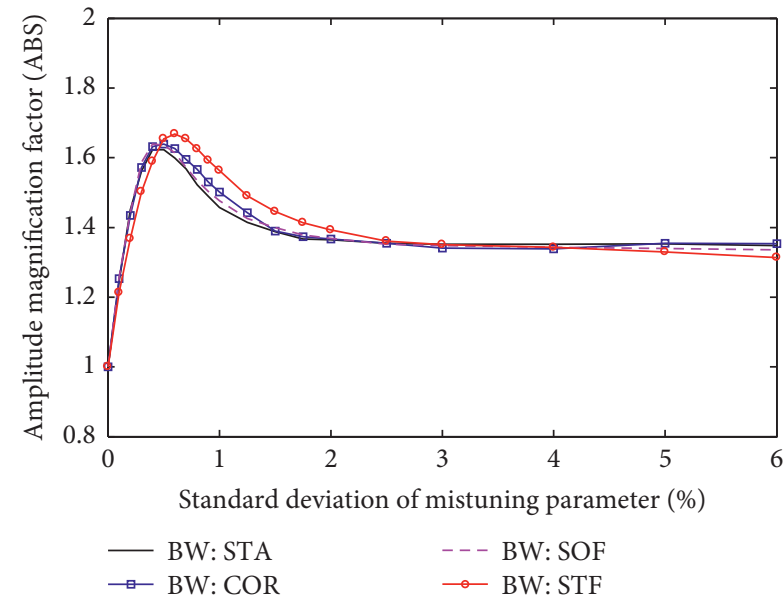

(b)

FIGURE 18: $95^{\text {th }}$ percentile of absolute AMF versus standard deviation of mistuning parameter under the action of (a) 3EO and (b) $4 \mathrm{EO}$ backward traveling wave excitations. Monte Carlo simulation of 2000 mistuning patterns generated by uniform distribution of mean 0 is conducted for each point.

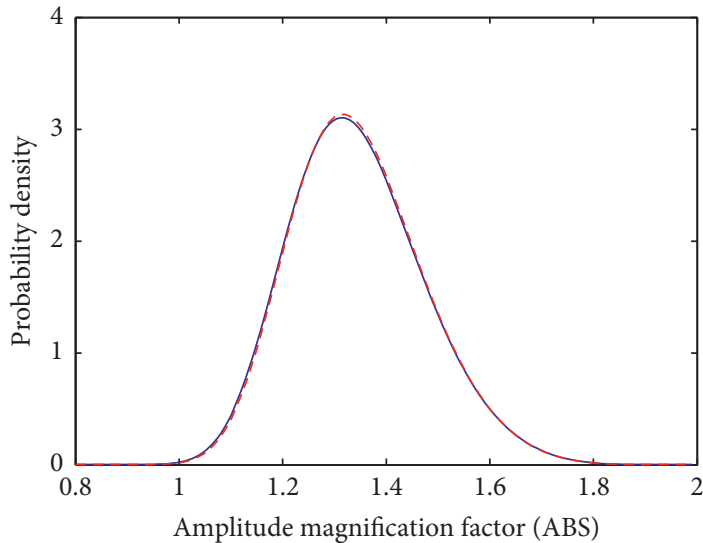

- FW: STA

-- BW: STA

(a)

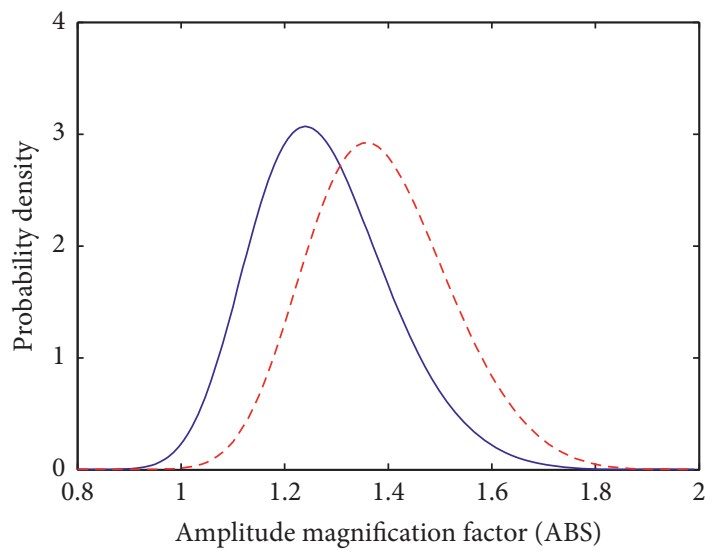

- FW: SOF

- - - BW:SOF

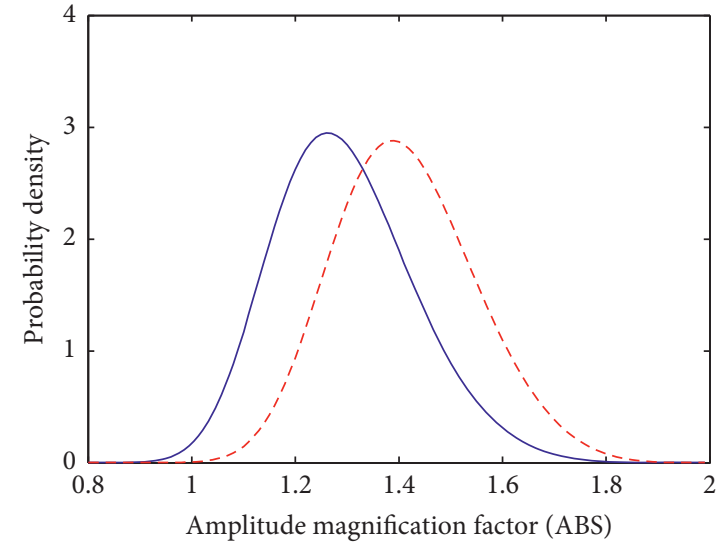

- FW: COR

- - BW: COR

(b)

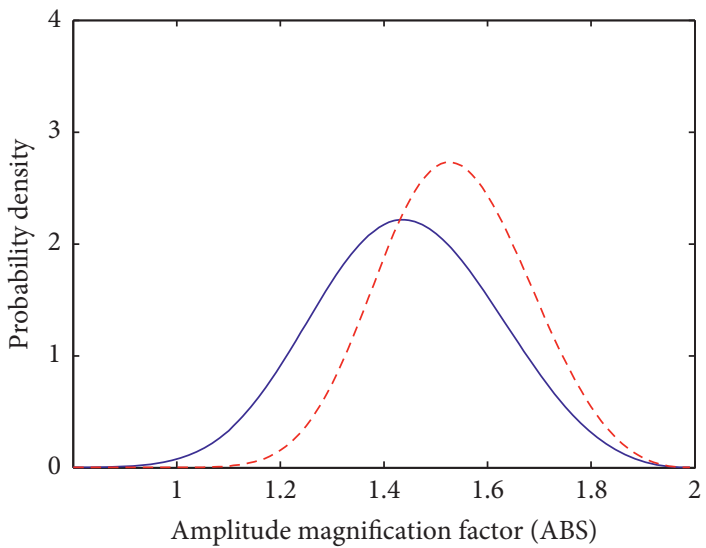

- FW: STF

- - - BW:STF

(c)

(d)

Figure 19: Comparison on the probability densities versus absolute AMF under the action of backward and forward traveling wave excitations of 3EO: (a) case STA, (b) case COR, (c) case SOF, and (d) case STF. 2000 mistuning patterns generated by uniform distribution of mean 0 and standard deviation $2.0 \%$ are used in Monte Carlo simulation. 
Finally, the effects of the direction of traveling wave excitation on the probability distribution of mistuned bladed disk are discussed. Figure 19 shows the comparison on the probability densities under the action of backward and forward traveling wave excitations of 3EO, where 2000 mistuning patterns generated by uniform distribution of mean 0 and standard deviation $2.0 \%$ are used. For the STA case, although the results in Figure 9(a) illustrate that the direction of traveling wave excitation may affect the forced response of mistuned bladed disk, its effect on the probability distribution of the absolute AMF is negligible, as shown in Figure 19(a). Nevertheless, the differences between the forward and backward ones are quite obvious for the COR, SOF and STF cases. It can be seen in Figures 19(b)-19(d) that the AMFs of backward ones tend to distribute over the ranges with larger values. Thus, for rotating bladed disk subjected to backward traveling wave excitations, which are the common cases in practical applications, the maximum cyclic stress that might encounter would be larger than subjecting to forward ones. The results shown in this section illustrate also, from a more comprehensive perspective, the remarkable influences of Coriolis forces, spin softening, and stress stiffening effects on the vibration of rotating bladed disks.

\section{Conclusions}

In this paper, the vibration characteristics of rotating mistuned bladed disks considering Coriolis forces, spin softening, and stress stiffening effects are addressed. Finite element models of bladed disks, combined with the effective component-mode mistuning method, are employed to generate compact and accurate reduced-order models. An efficient method for obtaining the stress stiffening matrix of the sector model is developed. On this basis, the models are used to study the influences of rotordynamic effects on the vibration of rotating bladed disk, with particular interest in their effects on the forced response and the statistical characteristics of responses. According to the results in this paper, some main conclusions are summarized as follows:

(1) The Coriolis force, spin softening, and stress stiffening effects have nonnegligible influences on the forced response of tuned and mistuned bladed disks, and the influences tend to be much more significant at the ranges of high rotating speed. All these three factors could lead to the shift of resonant frequency, where the influences caused by Coriolis force is relatively slight and those due to the spin softening and stress stiffening effects are more significant. The amplitudes of forced response, as well as the amplitude distributions between blades, can also be affected by the rotordynamic effects.
(2) The Coriolis force, spin softening, and stress stiffening effects have remarkable influences on the statistical characteristics of forced response of randomly mistuned bladed disk, where the probability distributions of the maximum response amplitude are obviously changed. Compared with the results of the case without considering rotordynamic effects, the spin softening effect tends to shift the probability distribution of maximum response amplitude towards higher values, whereas the stress stiffening effect plays a different role and decreases the values that the maximum amplitude distributes. Besides, the largest AMF of mistuned bladed disks in rotating conditions may be much larger than that predicted by the models without considering rotordynamic effects at some operating conditions.

(3) The extent of influence due to rotordynamic effects differs between different EOs of excitation due to their distinctions in rotating speed and the system's sensitiveness to the excitations. The direction of traveling wave excitation has also obvious effects on the forced response, the amplitude distribution between blades, and the statistical characteristics of responses.

The influences of Coriolis force, spin softening, and stress stiffening effects on the vibration of rotor systems have been widely appreciated in the field of rotordynamics. Nevertheless, their influences on the vibration of bladed disks are seldom reported in available investigations. In available studies, the rotordynamic effects tend to be assumed to be negligible during analysis. By the results shown in this paper, however, it can be seen that the rotordynamic effects could significantly affect the vibration of mistuning bladed disks, especially when the disks operate at high rotating speed. Thus, these rotordynamic effects should be well taken into account in the design of bladed disks.

\section{Appendix}

\section{Obtaining the Stress Stiffening Matrix Based on the Three-Sector Model}

In order to obtain the stress stiffening matrix of a cyclic sector model, an efficient method is developed using a threesector model of the bladed disk. Herein, a model with sectors $s-1, s$, and $s+1$ is employed for illustration. First, the degrees of freedom of each sector are divided into three parts regarding the upper boundary, interior part, and lower boundary, which are represented by symbols " $a$," " $i$," and " $b$ ", respectively. As common boundaries exist in the threesector model, the vector of response and the corresponding elastic stiffness matrix in the global Cartesian coordinate system can be expressed as 


$$
\begin{aligned}
\mathbf{u}_{T S} & =\left\{\begin{array}{c}
\mathbf{u}_{a}^{(s-1)} \\
\mathbf{u}_{i}^{(s-1)} \\
\mathbf{u}_{a}^{(s)} \\
\mathbf{u}_{i}^{(s)} \\
\mathbf{u}_{a}^{(s+1)} \\
\mathbf{u}_{i}^{(s+1)} \\
\mathbf{u}_{b}^{(s+1)}
\end{array}\right\}, \\
\mathbf{K}_{T S} & =\left[\begin{array}{llllll}
\mathbf{K}_{a a}^{(s-1)} & \mathbf{K}_{a i}^{(s-1)} & \mathbf{K}_{a b}^{(s-1)} \\
\mathbf{K}_{i a}^{(s-1)} & \mathbf{K}_{i i}^{(s-1)} & \mathbf{K}_{i b}^{(s-1)} & & & \\
\mathbf{K}_{b a}^{(s-1)} & \mathbf{K}_{b i}^{(s-1)} & \mathbf{K}_{b b}^{(s-1)}+\mathbf{K}_{a a}^{(s)} & \mathbf{K}_{a i}^{(s)} & \mathbf{K}_{a b}^{(s)} & \\
& & \mathbf{K}_{i a}^{(s)} & \mathbf{K}_{i i}^{(s)} & \mathbf{K}_{i b}^{(s)} & \\
& \mathbf{K}_{b a}^{(s)} & \mathbf{K}_{b i}^{(s)} & \mathbf{K}_{b b}^{(s)}+\mathbf{K}_{a a}^{(s+1)} & \mathbf{K}_{a i}^{(s+1)} & \mathbf{K}_{a b}^{(s+1)} \\
& & & \mathbf{K}_{i a}^{(s+1)} & \mathbf{K}_{i i}^{(s+1)} & \mathbf{K}_{i b}^{(s+1)} \\
& & & \mathbf{K}_{b a}^{(s+1)} & \mathbf{K}_{b i}^{(s+1)} & \mathbf{K}_{b b}^{(s+1)}
\end{array}\right] .
\end{aligned}
$$

The centrifugal forces acted on each sector are identical in their local cylindrical coordinate systems, where only the responses of 0EO types can be excited for a tuned bladed disk. During the analysis on a single cyclic sector, the $0 \mathrm{EO}$ type of cyclic-symmetric boundary conditions is applied by setting the responses on the upper and lower boundaries to be identical in cylindrical coordinate system. For the threesector model, the boundary conditions can be applied by constraining the response of the upper boundary of sector $s-1$ and that of the lower boundary of sector $s+1$ to be identical in the global cylindrical coordinate system. Such constraints are equivalent to the following coordinate transformation in global Cartesian coordinate system:

$$
\mathbf{u}_{T S}=\left\{\begin{array}{c}
\mathbf{u}_{a}^{(s-1)} \\
\mathbf{u}_{i}^{(s-1)} \\
\mathbf{u}_{a}^{(s)} \\
\mathbf{u}_{i}^{(s)} \\
\mathbf{u}_{a}^{(s+1)} \\
\mathbf{u}_{i}^{(s+1)} \\
\mathbf{u}_{b}^{(s+1)}
\end{array}\right\}=\left[\begin{array}{cccccc}
\mathbf{I}_{a} & \mathbf{0} & \mathbf{0} & \mathbf{0} & \mathbf{0} & \mathbf{0} \\
\mathbf{0} & \mathbf{I}_{i} & \mathbf{0} & \mathbf{0} & \mathbf{0} & \mathbf{0} \\
\mathbf{0} & \mathbf{0} & \mathbf{I}_{a} & \mathbf{0} & \mathbf{0} & \mathbf{0} \\
\mathbf{0} & \mathbf{0} & \mathbf{0} & \mathbf{I}_{i} & \mathbf{0} & \mathbf{0} \\
\mathbf{0} & \mathbf{0} & \mathbf{0} & \mathbf{0} & \mathbf{I}_{a} & \mathbf{0} \\
\mathbf{0} & \mathbf{0} & \mathbf{0} & \mathbf{0} & \mathbf{0} & \mathbf{I}_{i} \\
\mathbf{R}_{t} & \mathbf{0} & \mathbf{0} & \mathbf{0} & \mathbf{0} & \mathbf{0}
\end{array}\right]\left\{\begin{array}{c}
\mathbf{u}_{a}^{(s-1)} \\
\mathbf{u}_{i}^{(s-1)} \\
\mathbf{u}_{a}^{(s)} \\
\mathbf{u}_{i}^{(s)} \\
\mathbf{u}_{a}^{(s+1)} \\
\mathbf{u}_{a}^{(s+1)}
\end{array}\right\}=\widetilde{\mathbf{R}} \widetilde{\mathbf{u}}_{T S},
$$

where $\mathbf{I}_{a}$ and $\mathbf{I}_{i}$ are the identity matrices and $\mathbf{R}_{t}$ is the rotation transformation matrix between $\mathbf{u}_{b}^{(s+1)}$ and $\mathbf{u}_{a}^{(s-1)}$ and can be calculated by

$$
\mathbf{R}_{t}=\mathbf{I}_{t} \otimes \mathbf{R}_{e},
$$

where $\mathbf{I}_{t}$ is an identity matrix of dimension $N_{t}$, where $N_{t}$ denotes the number of degrees of nodes on the upper cyclic boundary, and $\mathbf{R}_{e}$ denotes an elementary rotation transformation matrix and

$$
\mathbf{R}_{e}=\left[\begin{array}{ccc}
\cos \theta_{e} & \sin \theta_{e} & 0 \\
-\sin \theta_{e} & \cos \theta_{e} & 0 \\
0 & 0 & 1
\end{array}\right],
$$

where $\theta_{e}$ denotes the relative angle between the upper boundary of sector $s-1$ and the lower boundary of sector $s+1$.

By applying the coordinate transformation in equation (A.2) and conducting static analysis under the action of centrifugal forces, the obtained stiffness matrix considering the stress stiffening effect can be expressed as

$$
\begin{aligned}
& \widetilde{\mathbf{K}}_{T S}=\left[\begin{array}{cccccc}
\widetilde{\mathbf{K}}_{a a}^{(s-1)}+\mathbf{R}_{t}^{\mathrm{T}} \widetilde{\mathbf{K}}_{b b}^{(s+1)} \mathbf{R}_{t} & \widetilde{\mathbf{K}}_{a i}^{(s-1)} & \widetilde{\mathbf{K}}_{a b}^{(s-1)} & \mathbf{0} & \mathbf{R}_{t}^{\mathrm{T}} \widetilde{\mathbf{K}}_{b a}^{(s+1)} & \mathbf{R}_{t}^{\mathrm{T}} \widetilde{\mathbf{K}}_{b i}^{(s+1)} \\
\widetilde{\mathbf{K}}_{i a}^{(s-1)} & \widetilde{\mathbf{K}}_{i i}^{(s-1)} & \widetilde{\mathbf{K}}_{i b}^{(s-1)} & \mathbf{0} & \mathbf{0} & \mathbf{0} \\
\widetilde{\mathbf{K}}_{b a}^{(s-1)} & \widetilde{\mathbf{K}}_{b i}^{(s-1)} & \widetilde{\mathbf{K}}_{b b}^{(s-1)}+\widetilde{\mathbf{K}}_{a a}^{(s)} & \widetilde{\mathbf{K}}_{a i}^{(s)} & \widetilde{\mathbf{K}}_{a b}^{(s)} & \mathbf{0} \\
\mathbf{0} & \mathbf{0} & \widetilde{\mathbf{K}}_{i a}^{(s)} & \widetilde{\mathbf{K}}_{i i}^{(s)} & \widetilde{\mathbf{K}}_{i b}^{(s)} & \mathbf{0} \\
\widetilde{\mathbf{K}}_{a b}^{(s+1)} \mathbf{R}_{t} & \mathbf{0} & \widetilde{\mathbf{K}}_{b a}^{(s)} & \widetilde{\mathbf{K}}_{b i}^{(s)} & \widetilde{\mathbf{K}}_{b b}^{(s)}+\widetilde{\mathbf{K}}_{a a}^{(s+1)} & \widetilde{\mathbf{K}}_{a i}^{(s+1)} \\
\widetilde{\mathbf{K}}_{i b}^{(s+1)} \mathbf{R}_{t} & \mathbf{0} & \mathbf{0} & \mathbf{0} & \widetilde{\mathbf{K}}_{i a}^{(s+1)} & \widetilde{\mathbf{K}}_{i i}^{(s+1)}
\end{array}\right] . \\
& \begin{aligned}
\mathbf{K}_{s, 0}^{s} & =\left[\begin{array}{cc}
\widetilde{\mathbf{K}}_{b b}^{(s-1)}+\widetilde{\mathbf{K}}_{a a}^{(s)} & \widetilde{\mathbf{K}}_{a i}^{(s)} \\
\widetilde{\mathbf{K}}_{i a}^{(s)} & \widetilde{\mathbf{K}}_{i i}^{(s)}
\end{array}\right], \\
\mathbf{K}_{s, 1}^{s} & =\left[\begin{array}{cc}
\widetilde{\mathbf{K}}_{a b}^{(s)} \mathbf{R}_{s} & \mathbf{0} \\
\widetilde{\mathbf{K}}_{i b}^{(s)} \mathbf{R}_{s} & \mathbf{0}
\end{array}\right]
\end{aligned}
\end{aligned}
$$

As can be seen in the matrix, the block matrices in the third and fourth rows are not affected by the coordinate transformation. As the above analysis is conducted in the global Cartesian coordinate system, some further disposals are needed to obtain the acquired matrices of a sector model as shown in equation (10). Finally, the acquired stiffness matrices considering the stress stiffening effects can be obtained by the block matrices of $\widetilde{\mathbf{K}}_{T S}$ as where $\mathbf{R}_{s}$ denotes a rotation transformation matrix and 


$$
\begin{aligned}
\mathbf{R}_{s} & =\mathbf{I}_{t} \otimes \mathbf{R}_{c}, \\
\mathbf{R}_{c} & =\left[\begin{array}{ccc}
\cos \theta_{c} & \sin \theta_{c} & 0 \\
-\sin \theta_{c} & \cos \theta_{c} & 0 \\
0 & 0 & 1
\end{array}\right],
\end{aligned}
$$

where $\theta_{c}$ denotes the relative angle between the upper and lower boundaries of sector $s$.

The rotation transformation matrix $\mathbf{R}_{s}$ is included in equation (A.6) because the cyclic boundary conditions are defined with respect to the coordinates in the cylindrical coordinate system, whereas the matrices are obtained in a Cartesian one. Thus, appropriate coordinate transformation is needed. As statics analysis is conducted, the resulting stiffness matrices contain simultaneously the contributions of elastic stiffness and stress stiffening effects. Then, the spin softening matrix, which can be directly obtained by the sector model, should be added to acquire the overall stiffness matrix of a rotating bladed disk.

\section{Data Availability}

The data used to support the findings of this study are available from the corresponding author upon request.

\section{Conflicts of Interest}

The authors declare that there are no conflicts of interest regarding the publication of this paper.

\section{Acknowledgments}

This work was supported by the Project of National Natural Science Foundation of China (No. 51805130), China Postdoctoral Science Foundation Funded Project (No. 2018M632521), and Natural Science Foundation of Anhui Province (No. 1808085QE138).

\section{References}

[1] J. C. Slater, G. R. Minkiewicz, and A. J. Blair, "Forced response of bladed disk assemblies-a survey," The Shock and Vibration Digest, vol. 31, no. 1, pp. 17-24, 1999.

[2] M. P. Castanier and C. Pierre, "Modeling and analysis of mistuned bladed disk vibration: current status and emerging directions," Journal of Propulsion and Power, vol. 22, no. 2, pp. 384-396, 2006.

[3] J. Yuan, F. Scarpa, G. Allegri, B. Titurus, S. Patsias, and R. Rajasekaran, "Efficient computational techniques for mistuning analysis of bladed discs: a review," Mechanical Systems and Signal Processing, vol. 87, pp. 71-90, 2017.

[4] D. J. Ewins and Y. J. Chan, Vibration of Rotating Bladed Discs: Mistuning, Coriolis, and Robust Design, Springer Netherlands, Dordrecht, Netherlands, 2011.

[5] D. J. Ewins and Z. S. Han, "Resonant vibration levels of a mistuned bladed disk," Journal of Vibration and Acoustics, vol. 106, no. 2, pp. 211-217, 1984.

[6] S.-T. Wei and C. Pierre, "Localization phenomena in mistuned assemblies with cyclic symmetry part II: forced vibrations," Journal of Vibration and Acoustics, vol. 110, no. 4, pp. 439-449, 1988.
[7] A. Sinha and S. Chen, "A higher order technique to compute the statistics of forced response of a mistuned bladed disk assembly," Journal of Sound and Vibration, vol. 130, no. 2, pp. 207-221, 1989.

[8] C. C. Lin and M. P. Mignolet, "Effects of damping and damping mistuning on the forced vibration response of bladed disks," Journal of Sound and Vibration, vol. 193, pp. 525-543, 1993.

[9] B. C. Watson, M. P. Kamat, and D. V. Murthy, "Forced response of mistuned bladed disk assemblies," in Proceedings of the AIAA/ASME/ASCE/AHS/ASC Structures, Structural Dynamics, \& Materials Conference, La Jolla, CA, USA, 1993.

[10] P. A. Borea, K. Varani, A. Dalpiaz, A. Capuzzo, E. Fabbri, and A. P. Ijzerman, "Vibration and flutter of mistuned bladed-disk assemblies," Journal of Propulsion and Power, vol. 1, pp. 336-344, 1984.

[11] R. E. Kielb and K. R. V. Kaza, "Effects of structural coupling on mistuned cascade flutter and response," Journal of Engineering for Gas Turbines and Power, vol. 106, no. 1, pp. 17-24, 1984.

[12] J. Chen, Q. Chen, D. Shen, and F. Fan, "Effects of blade mistuning and blade-disk on cascade flutter boundaries," Acta Aeronautica et Astronautica Sinica, vol. 9, no. 8, 1988.

[13] C. Martel, R. Corral, and J. M. Llorens, "Stability increase of aerodynamically unstable rotors using intentional mistuning," Journal of Turbomachinery, vol. 130, 2007.

[14] Z. He, B. I. Epureanu, and C. Pierre, "Parametric study of the aeroelastic response of mistuned bladed disks," Computers \& Structures, vol. 85, no. 11-14, pp. 852-865, 2007.

[15] Y. S. Choi, D. A. Gottfried, and S. Fleeter, "Resonant response of mistuned bladed disks including aerodynamic damping effects," Journal of Propulsion and Power, vol. 26, no. 1, pp. 16-24, 2010.

[16] E. P. Petrov, "A method for forced response analysis of mistuned bladed disks with aerodynamic effects included," Journal of Engineering for Gas Turbines and Power, vol. 132, 2010.

[17] B. Shapiro and K. E. Willcox, "Analyzing mistuning of bladed disks by symmetry and reduced-order areodynamic modeling," Journal of Propulsion and Power, vol. 19, pp. 307-311, 2015.

[18] C. Martel and J. J. Sánchez-Álvarez, "Maximum mistuning amplification of the forced response vibration of turbomachinery rotors in the presence of aerodynamic damping," Journal of Sound and Vibration, vol. 397, pp. 108-122, 2017.

[19] S. Willeke, L. Schwerdt, L. Panning-von Scheidt, and J. Wallaschek, "Intentional response reduction by harmonic mistuning of bladed disks with aerodynamic damping," Journal of Engineering for Gas Turbines and Power, vol. 140, 2018.

[20] M. Castanier and C. Pierre, "Investigation of the combined effects of intentional and random mistuning on the forced response of bladed disks," in Proceedings of the 34th AIAA/ ASME/SAE/ASEE Joint Propulsion Conference and Exhibit, American Institute of Aeronautics and Astronautics, Cleveland, OH, USA, July 1998.

[21] R. Bladh, M. P. Castanier, and C. Pierre, "Component-modebased reduced order modeling techniques for mistuned bladed disks: part I - theoretical models," Journal of Engineering for Gas Turbines and Power, vol. 123, no. 1, pp. 89-99, 2000.

[22] R. Bladh, Efficient Predictions of the Vibratory Response of Mistuned Bladed Disks by Reduced Order Modeling, The University of Michigan, Ann Arbor, MI, USA, 2001. 
[23] M.-T. Yang and J. H. Griffin, "A reduced-order model of mistuning using a subset of nominal system modes," Journal of Engineering for Gas Turbines and Power, vol. 123, no. 4, pp. 893-900, 2001.

[24] D. M. Feiner and J. H. Griffin, "A fundamental model of mistuning for a single family of modes," Journal of Turbomachinery, vol. 124, no. 4, pp. 597-605, 2002.

[25] S. H. Lim, R. Bladh, M. P. Castanier, and C. Pierre, "Compact, generalized component mode mistuning representation for modeling bladed disk vibration," AIAA Journal, vol. 45, pp. 2285-2298, 2003.

[26] A. Sternchüss, Multi-Level Parametric Reduced Models of Rotating Bladed Disk Assemblies, Ecole Centrale Paris, Châtenay-Malabry, France, 2009.

[27] J. A. Beck, J. M. Brown, C. J. Cross, and J. C. Slater, "Component-mode reduced-order models for geometric mistuning of integrally bladed rotors," AIAA Journal, vol. 52, no. 7, pp. 1345-1356, 2014.

[28] P. Vargiu, C. M. Firrone, S. Zucca, and M. M. Gola, "A reduced order model based on sector mistuning for the dynamic analysis of mistuned bladed disks," International Journal of Mechanical Sciences, vol. 53, no. 8, pp. 639-646, 2011.

[29] K. D’Souza, C. Jung, and B. I. Epureanu, "Analyzing mistuned multi-stage turbomachinery rotors with aerodynamic effects," Journal of Fluids and Structures, vol. 42, pp. 388-400, 2013.

[30] B. W. Huang and J. H. Kuang, "Mode localization in a rotating mistuned turbo disk with Coriolis effect," International Journal of Mechanical Sciences, vol. 43, no. 7, pp. 1643-1660, 2001.

[31] M. Nikolic, E. P. Petrov, and D. J. Ewins, "Coriolis forces in forced response analysis of mistuned bladed disks," Journal of Turbomachinery, vol. 129, no. 4, pp. 730-739, 2006.

[32] X. Kan, Z. Xu, B. Zhao, and J. Zhong, "Effect of coriolis force on forced response magnification of intentionally mistuned bladed disk," Journal of Sound and Vibration, vol. 399, pp. 124-136, 2017.

[33] E. Chatelet, F. D'Ambrosio, and G. Jacquet-Richardet, "Toward global modelling approaches for dynamic analyses of rotating assemblies of turbomachines," Journal of Sound and Vibration, vol. 282, no. 1-2, pp. 163-178, 2005.

[34] J. Li, M. Castanier, C. Pierre, and S. Ceccio, "Experimental monte carlo mistuning assessment of bladed disk vibration using forcing variations," in Proceedings of the 47th AIAA/ ASME/ASCE/AHS/ASC Structures, Structural Dynamics, and Materials Conference, American Institute of Aeronautics and Astronautics, Newport, RI, USA, May 2006. 


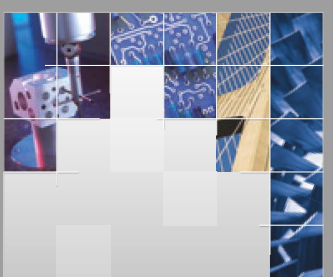

\section{Enfincering}
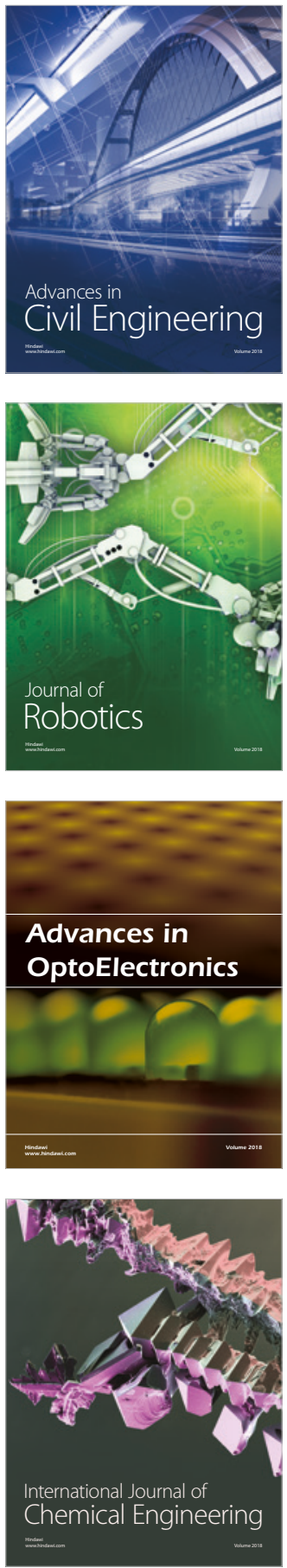

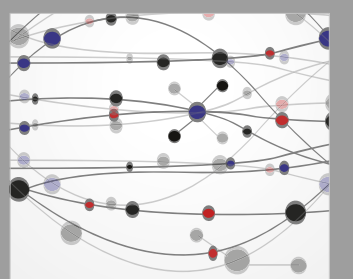

\section{Rotating \\ Machinery}

The Scientific World Journal

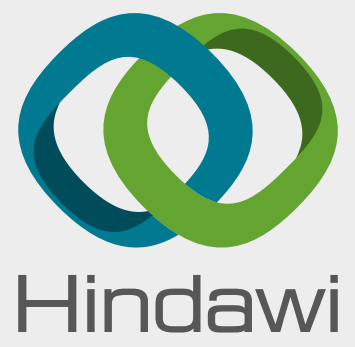

Submit your manuscripts at

www.hindawi.com
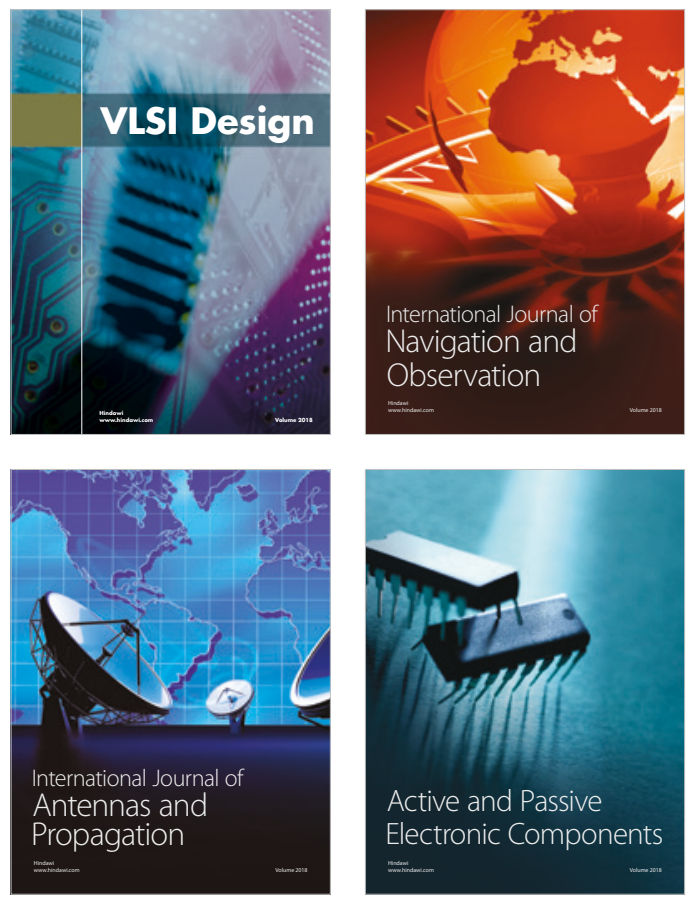
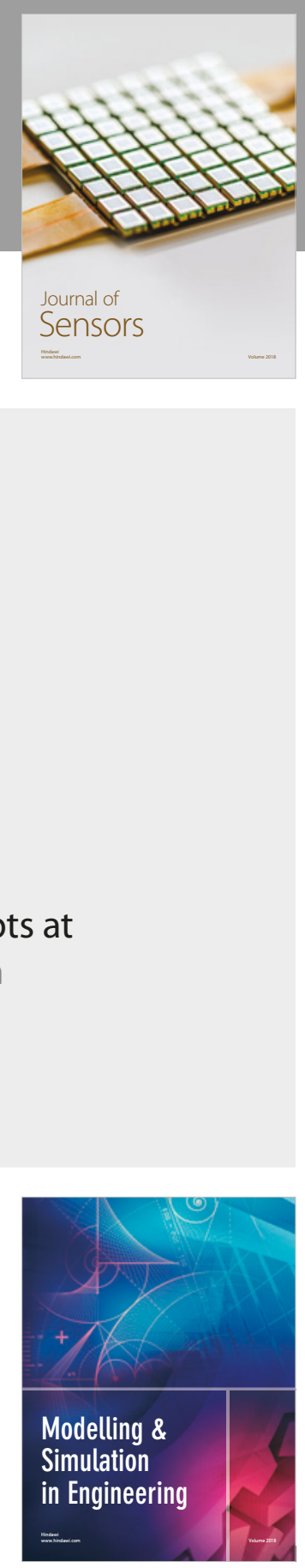

\section{Advances \\ Multimedia}
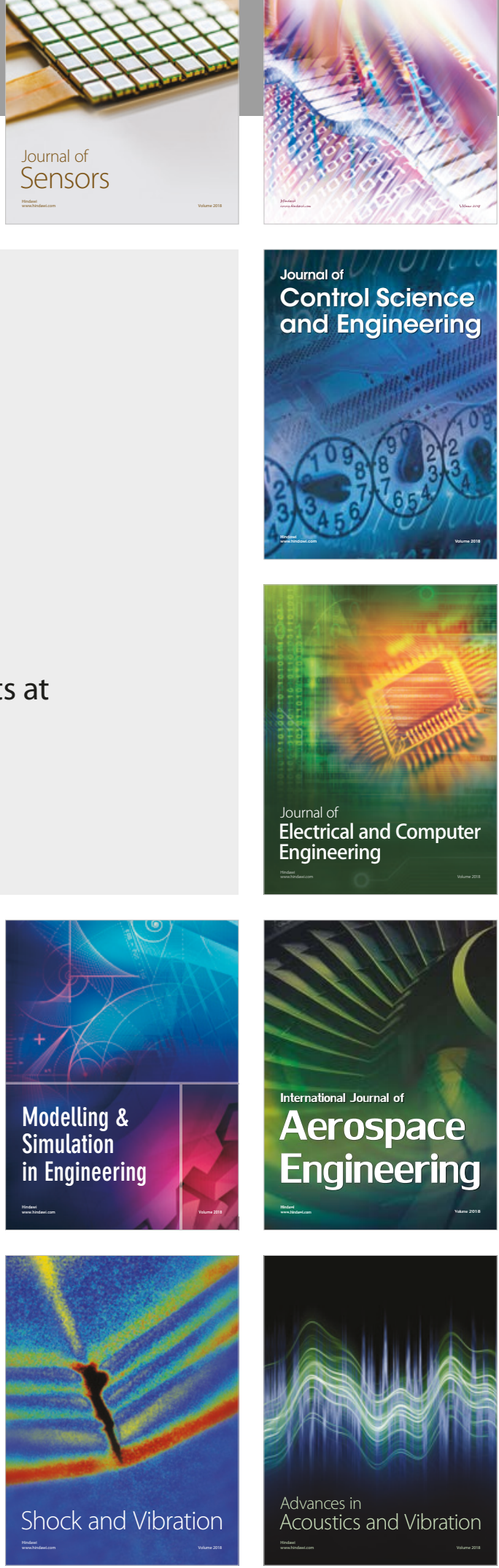